\title{
Output Feedback Adaptive Control of Non-Minimum Phase Systems Using Optimal Control Modification
}

\author{
Nhan Nguyen* \\ NASA Ames Research Center, Moffett Field, CA 94035 \\ Kelley E. Hashemi ${ }^{\dagger}$ \\ Universities Space Research Association, Moffett Field, CA 94035 \\ Tansel Yucelen $\ddagger$ \\ University of South Florida, FL 33620 \\ Ehsan Arabi ${ }^{\S}$ \\ University of South Florida, FL 33620
}

\begin{abstract}
This paper describes output feedback adaptive control approaches for non-minimum phase SISO systems with relative degree 1 and non-strictly positive real (SPR) MIMO systems with uniform relative degree 1 using the optimal control modification method. It is well-known that the standard model-reference adaptive control (MRAC) cannot be used to control non-SPR plants to track an ideal SPR reference model. Due to the ideal property of asymptotic tracking, MRAC attempts an unstable pole-zero cancellation which results in unbounded signals for non-minimum phase SISO systems. The optimal control modification can be used to prevent the unstable pole-zero cancellation which results in a stable adaptation of non-minimum phase SISO systems. However, the tracking performance using this approach could suffer if the unstable zero is located far away from the imaginary axis. The tracking performance can be recovered by using an observer-based output feedback adaptive control approach which uses a Luenberger observer design to estimate the state information of the plant. Instead of explicitly specifying an ideal SPR reference model, the reference model is established from the linear quadratic optimal control to account for the non-minimum phase behavior of the plant. With this non-minimum phase reference model, the observer-based output feedback adaptive control can maintain stability as well as tracking performance. However, in the presence of the mismatch between the SPR reference model and the non-minimum phase plant, the standard MRAC results in unbounded signals, whereas a stable adaptation can be achieved with the optimal control modification. An application of output feedback adaptive control for a flexible wing aircraft illustrates the approaches.
\end{abstract}

\section{Introduction}

Consider the system

$$
\begin{gathered}
\dot{x}=A x+B u+\Delta(x, z, u) \\
\dot{z}=f(x, z, u) \\
y=C x
\end{gathered}
$$

where $z(t)$ is an internal state vector, $\Delta(x, z, u)$ is the plant model error that is unknown and not accounted for, $\dot{z}(t)$ is the unmodeled dynamics, and $y(t)$ is the plant output vector.

When the tracking error based on only the output signal $y(t)$ is used for model-reference adaptive control (MRAC), such a class of adaptive control is called output feedback adaptive control. If a stable reference model $y_{m}(t)$ is specified for the output $y(t)$ to track, then model-reference adaptive control is feasible if the plant transfer function satisfies the strictly positive real (SPR) condition. Therefore, an essential requirement for output feedback adaptive control is the

\footnotetext{
*Technical Group Lead, Associate Fellow AIAA, Intelligent Systems Division, nhan.t.nguyen@nasa.gov

${ }^{\dagger}$ NASA Post-Doctoral Fellow, Intelligent Systems Division, kelley.e.hashemi@ nasa.gov

$\ddagger$ Assistant Professor and Director of the Laboratory for Autonomy, Control, Information, and Systems, yucelen@lacis.team

${ }^{\S}$ Graduate Research Assistant, ehsanarabi@mail.usf.edu
} 
SPR condition which the transfer function must satisfy. Classical treatments of output feedback adaptive control are discussed extensively by Åström and Wittenmark [1], Ioannu [2], and Narendra and Annaswamy [3].

Non-minimum phase systems, on the other hand, do not possess SPR transfer functions. Therefore, output feedback adaptive control of systems with non-SPR transfer functions can be challenging. Non-minimum phase plants generally pose more difficulty to MRAC than minimum phase plants. Output feedback adaptive control generally relies on the SPR property to ensure stability. The difficulty with adaptive control design for non-minimum phase plants is due to the ideal property of MRAC which attempts to seek asymptotic tracking at all cost. This results in a pole-zero cancellation in the right half plane for non-minimum phase systems which leads to unbounded signals [4].

The linear asymptotic property of the optimal control modification [5] can be used for adaptive control of nonminimum phase plants [4]. By modifying the standard MRAC, the pole-zero cancellation in the right half plane can be prevented. This then results in bounded tracking as opposed to asymptotic tracking. Closed-loop stability can be obtained by a judicious choice of the modification parameter $v$. However, the tracking performance of this approach could suffer if the unstable zero is located far away from the imaginary axis. The tracking performance can be recovered by using an observer-based output feedback adaptive control approach which uses a Luenberger observer design to estimate the state information of the plant. Instead of explicitly specifying an ideal SPR reference model, the reference model is established from the linear quadratic optimal control to account for the non-minimum phase behavior of the plant. With this non-minimum phase reference model, the observer-based output feedback adaptive control can maintain stability as well as tracking performance. It should be noted that Lavretsky and Wise also develop an observer-based output feedback adaptive control approach with a loop transfer recovery [6]. Lavretsky further extends this method for non-minimum phase plants with arbitrary relative degree [7].

In this paper, we will present an optimal control modification output feedback adaptive control method for nonminimum phase single-input single-out (SISO) systems with relative degree 1. Bounded tracking is guaranteed with this method. The optimal control modification output feedback adaptive control method is then extended to non-SPR multiple input multiple output (MIMO) systems with uniform relative degree 1. Finally, an observer-based output feedback adaptive control method is developed for non-SPR MIMO systems using the optimal control modification. The study shows that the optimal control modification can improve stability and performance of output feedback adaptive control systems in all cases.

\section{Output Feedback Adaptive Control of Non-Minimum Phase SISO Sys- tems with Relative Degree 1}

Consider the following SISO plant:

$$
\begin{aligned}
\dot{x}=A x+B u & \Leftrightarrow\left[\begin{array}{c}
\dot{x}_{1} \\
\dot{x}_{2}
\end{array}\right]=\left[\begin{array}{ll}
a & g \\
l & h
\end{array}\right]\left[\begin{array}{l}
x_{1} \\
x_{2}
\end{array}\right]+\left[\begin{array}{c}
b \\
m
\end{array}\right] u \\
y & =C x=\left[\begin{array}{ll}
1 & 0
\end{array}\right]\left[\begin{array}{l}
x_{1} \\
x_{2}
\end{array}\right]=x_{1}
\end{aligned}
$$

with $x(0)=x_{0}$, where $x_{2}(t)$ is the unmeasurable state with internal dynamics, $a$ unknown but all the other parameters are known, $b \neq 0$, and $h<0$.

The transfer functions of the plant is expressed as

$$
\frac{y}{u}=W_{p}(s)=k_{p} \frac{Z_{p}(s)}{R_{p}(s)}=\frac{b(s-h)+g m}{(s-a)(s-h)-g l}
$$

where $k_{p}=b$. Note that $W_{m}(s)$ is SPR with a relative degree 1 . The plant also has a relative degree 1 and is assumed to be stable. So, $R_{p}(s)=(s-a)(s-h)-g l$ is Hurwitz.

The objective is to design an output feedback adaptive controller to track the following reference model:

$$
y_{m}=W_{m}(s) r=k_{m} \frac{Z_{m}(s)}{R_{m}(s)} r=\frac{b_{m} r}{s-a_{m}}
$$

with $y_{m}(0)=y_{m_{0}}$, where $a_{m}<0$ and $k_{m}=b_{m}$. The ideal output feedback adaptive controller is designed to be of the form

$$
u^{*}=-b^{-1} \theta_{1}^{*} y^{*}-\frac{b^{-1} \theta_{2}^{*} y^{*}}{s-\lambda}-\frac{b^{-1} \theta_{3}^{*} u^{*}}{s-\lambda}+b^{-1} b_{m} r
$$


where $\lambda<0$ is a chosen parameter, and $\theta_{1}^{*}, \theta_{2}^{*}$, and $\theta_{3}^{*}$ are unknown constants.

$u^{*}(s)$ is obtained explicitly as

$$
u^{*}=\frac{b^{-1}\left[-\theta_{1}^{*}(s-\lambda)-\theta_{2}^{*}\right] y^{*}+b^{-1} b_{m}(s-\lambda) r}{s-\lambda+b^{-1} \theta_{3}^{*}}
$$

Then, the ideal output $y^{*}(s)$ is written as

$$
y^{*}=\frac{Z_{p}(s)}{R_{p}(s)} \frac{\left[-\theta_{1}^{*}(s-\lambda)-\theta_{2}^{*}\right] y^{*}+b_{m}(s-\lambda) r}{s-\lambda+b^{-1} \theta_{3}^{*}}
$$

We consider the following cases: 1) the plant is minimum phase, and 2) the plant is non-minimum phase.

\subsection{Minimum Phase Plant}

If the plant is minimum phase, then $Z_{p}(s)$ is Hurwitz. Then, the pole-zero cancellation can take place in the left half plane. Therefore, it follows that

$$
Z_{p}(s)=s-\lambda+b^{-1} \theta_{3}^{*}
$$

This results in

$$
\theta_{3}^{*}=b(\lambda-h)+g m
$$

The ideal controller $u^{*}(s)$ then becomes

$$
u^{*}=\frac{b^{-1}\left[-\theta_{1}^{*}(s-\lambda)-\theta_{2}^{*}\right] y^{*}+b^{-1} b_{m}(s-\lambda) r}{Z_{p}(s)}
$$

The ideal output $y^{*}(s)$ is then obtained as

$$
y^{*}=\frac{\left[-\theta_{1}^{*}(s-\lambda)-\theta_{2}^{*}\right] y^{*}+b_{m}(s-\lambda) r}{(s-a)(s-h)-g l}
$$

This results in the following ideal closed-loop transfer function:

$$
\frac{y^{*}}{r}=\frac{b_{m}(s-\lambda)}{s^{2}-\left(a+h-\theta_{1}^{*}\right) s+a h-g l-\lambda \theta_{1}^{*}+\theta_{2}^{*}}
$$

We want the ideal closed-loop plant to track the reference model. So, the ideal closed-loop transfer function must be equal to the reference model transfer function $W_{m}(s)$. Thus,

$$
\frac{b_{m}(s-\lambda)}{s^{2}-\left(a+h-\theta_{1}^{*}\right) s+a h-g l-\lambda \theta_{1}^{*}+\theta_{2}^{*}}=\frac{b_{m}}{s-a_{m}}
$$

This leads to the following model matching conditions:

$$
\begin{gathered}
a+h-\theta_{1}^{*}=\lambda+a_{m} \\
a h-g l-\lambda \theta_{1}^{*}+\theta_{2}^{*}=\lambda a_{m}
\end{gathered}
$$

$\theta_{1}^{*}$ and $\theta_{2}^{*}$ are then obtained as

$$
\begin{gathered}
\theta_{1}^{*}=a-a_{m}+h-\lambda \\
\theta_{2}^{*}=g l-a h+\lambda(a+h-\lambda)
\end{gathered}
$$

The adaptive controller is now established as

$$
u=-b^{-1} \theta_{1} y-\frac{b^{-1} \theta_{2} y}{s-\lambda}-\frac{b^{-1} \theta_{3} u}{s-\lambda}+b^{-1} b_{m} r
$$


where $\theta_{1}(t), \theta_{2}(t)$, and $\theta_{3}(t)$ are the estimates of $\theta_{1}^{*}, \theta_{2}^{*}$, and $\theta_{3}^{*}$, respectively. Let $\tilde{\theta}_{1}(t)=\theta_{1}(t)-\theta_{1}^{*}, \tilde{\theta}_{2}(t)=$ $\theta_{2}(t)-\theta_{2}^{*}$, and $\tilde{\theta}_{3}(t)=\theta_{3}(t)-\theta_{3}^{*}$. Then, the output $y(s)$ of the closed-loop system is expressed as

$$
y=W_{m}(s) r-\frac{W_{m}(s)}{b_{m}}\left(\tilde{\theta}_{1} y+\frac{\tilde{\theta}_{2} y}{s-\lambda}+\frac{\tilde{\theta}_{3} u}{s-\lambda}\right)
$$

Define the tracking error as $e_{y}(t)=y_{m}(t)-y(t)$. Then, the tracking error equation is obtained as

$$
\dot{e}_{y}=a_{m} e_{y}+\tilde{\Theta}^{\top} \Phi(t)
$$

where $\tilde{\Theta}(t)=\left[\begin{array}{lll}\tilde{\theta}_{1}(t) & \tilde{\theta}_{2}(t) & \tilde{\theta}_{3}(t)\end{array}\right]^{\top}$ and $\Phi(t)=\left[\begin{array}{lll}\phi_{1}(t) & \phi_{2}(t) & \phi_{3}(t)\end{array}\right]^{\top}$ with

$$
\begin{gathered}
\phi_{1}=y \\
\dot{\phi}_{2}=\lambda \phi_{2}+y \\
\dot{\phi}_{3}=\lambda \phi_{3}+u
\end{gathered}
$$

Theorem 1 : The adaptive law given by

$$
\dot{\Theta}=-\Gamma \Phi(t) e_{y}
$$

with $\Gamma=\Gamma^{\top}>0$ is stable for the minimum phase plant in Eq. (79) and results in asymptotic tracking.

Proof: Since both the plant and reference model are minimum phase with the same relative degree 1, the closedloop poles are stable which implies $\Phi(t)$ is bounded. Suppose $\Phi(t)$ is bounded. We choose the Lyapunov candidate function

$$
V\left(e_{y}, \tilde{\Theta}\right)=e_{y}^{2}+\tilde{\Theta}^{\top} \Gamma^{-1} \tilde{\Theta}
$$

Then,

$$
\dot{V}\left(e_{y}, \tilde{\Theta}\right)=a_{m} e_{y}^{2} \leq 0
$$

Therefore, $e_{y}(t) \in \mathscr{L}_{2} \cap \mathscr{L}_{\infty}$ and $\tilde{\Theta}(t) \in \mathscr{L}_{\infty}$ are bounded. This implies $y(t), u(t)$, and $\Phi(t)$ are bounded. Note that since $\ddot{V}\left(e_{y}, \tilde{\Theta}\right)$ is bounded since $\Phi(t)$ is bounded. Therefore, $\dot{V}\left(e_{y}, \tilde{\Theta}\right)$ is uniformly continuous. Invoking the Barbalat's lemma, it follows that the output tracking error tends to zero asymptotically with $e_{y}(t) \rightarrow 0$ as $t \rightarrow \infty$.

\subsection{Non-Minimum Phase Plant}

For non-minimum phase plants, because the standard MRAC attempts to seek asymptotic tracking by performing a pole-zero cancellation in the right half plane, the resulting adaptive controller will become unbounded. Thus, if the adaptive law can be modified to seek only bounded tracking instead of asymptotic tracking, then this would prevent a pole-zero cancellation in the right half plane. The plant then can be stabilized.

For non-minimum phase plants, we consider two possible adaptive controllers.

1. We use the same adaptive controller in Eq. (18), but with the optimal control modification adaptive law

$$
\dot{\Theta}=-\Gamma \Phi(t)\left[e_{y}-v \Phi^{\top}(t) \Theta a_{m}^{-1}\right]
$$

By invoking the linear asymptotic property of the optimal control modification as $\Gamma \rightarrow \infty$ [4], we get

$$
\Theta^{\top} \Phi(t)=\frac{a_{m}\left(y_{m}-y\right)}{v}
$$

Then, the asymptotic linear controller tends to

$$
u=\frac{a_{m} y}{v b}+\frac{\left[v b_{m}-a_{m} W_{m}(s)\right] r}{v b}
$$

Comparing the ideal controller with the asymptotic linear controller, we see that the adaptive controller does not attempt to cancel the unstable zero of $W_{p}(s)$ since it has a stable pole at $s=a_{m}$ due to the term $W_{m}(s)$, as shown by the asymptotic closed-loop transfer function

$$
\frac{y}{r}=\frac{W_{p}(s)\left[v b_{m}-a_{m} W_{m}(s)\right]}{v b-a_{m} W_{p}(s)}
$$


Otherwise, the pole-zero cancellation would take place in the right half plane since $Z_{p}(s)$ is unstable. Therefore, the stability of the adaptive controller is no longer affected by the non-minimum phase behavior of $W_{p}(s)$. The stability of the closed-loop plant is then determined by a proper selection of the modification parameter $v$ such that $v>0$ and the closed-loop transfer function is stable. As $\Gamma \rightarrow \infty$, the equilibrium value of $y(t)$ tends to

$$
\bar{y}=\frac{W_{p}(0)\left[v b_{m}-a_{m} W_{m}(0)\right]}{v b-a_{m} W_{p}(0)} r
$$

Thus, the tracking is bounded regardless whether or not $W_{p}(s)$ is minimum phase. The closed-loop plant therefore is robustly stable with the optimal control modification. However, poor tracking will result if $v$ is too large to guarantee the closed-loop stability.

2. We use a simple adaptive controller for the first-order SISO plant as if the non-minimum phase dynamics do not exist with $x_{2}(t)=0$

$$
u(t)=k_{y}(t) y+k_{r} r
$$

where $k_{r}=b^{-1} b_{m}$ and $k_{y}(t)$ is computed by the optimal control modification adaptive law

$$
\dot{k}_{y}=\gamma_{y} y\left(e_{y}+v y k_{y} b a_{m}^{-1}\right) b
$$

with $\gamma_{y}>0$. By invoking the linear asymptotic property of the optimal control modification, we get

$$
k_{y} y=\frac{a_{m}\left(y-y_{m}\right)}{v b}
$$

Then, the asymptotic linear controller tends to

$$
u=\frac{a_{m} y}{v b}+\frac{\left[v b_{m}-a_{m} W_{m}(s)\right] r}{v b}
$$

Note that this asymptotic linear controller is the same as that with the first adaptive controller. Thus, even though the adaptive controllers are different, the closed-loop plants for both controllers behave the same in the limit.

We now will formalize the proof of the optimal control modification for the non-minimum phase plant (2) with relative degree 1 with $a$ unknown but all the other parameters are known and $h<0$. The adaptive controller is given by Eqs. (32) and (33).

The closed-loop plant becomes

$$
\begin{gathered}
\dot{y}=a_{m} y+b_{m} r+b \tilde{k}_{y} y+g z \\
\dot{z}=h z+\left(l+m k_{y}^{*}\right) y+m \tilde{k}_{y} y+m k_{r} r
\end{gathered}
$$

where $\tilde{k}_{y}(t)=k_{y}(t)-k_{y}^{*}$ and $k_{y}^{*}=\frac{a_{m}-a}{b}$.

The output tracking error equation is given by

$$
\dot{e}_{y}=a_{m} e_{y}-b \tilde{k}_{y} y-g z
$$

We define the reference internal state dynamics as

$$
\dot{z}_{m}=h z_{m}+\left(l+m k_{y}^{*}\right) y_{m}+m k_{r} r
$$
by

Let $e_{z}(t)=z_{m}(t)-z(t)$ be the internal state tracking error. Then, the internal state tracking error equation is given

$$
\dot{e}_{z}=h e_{z}+\left(l+m k_{y}^{*}\right) e_{y}-m \tilde{k}_{y} y
$$

Theorem 2: The non-minimum phase system (2) can be stabilized by the optimal control modification adaptive law (33) with a proper selection of the modification parameter $v$ that results in uniform ultimate boundedness of the tracking error.

Proof: Choose a Lyapunov candidate function

$$
V\left(e_{y}, e_{z}, \tilde{\theta}\right)=\alpha e_{y}^{2}+\beta e_{z}^{2}+\alpha \gamma_{y}^{-1} \tilde{k}_{y}^{2}
$$


where $\alpha>0$ and $\beta>0$.

$\dot{V}\left(e_{y}, e_{z}, \tilde{\theta}\right)$ is evaluated as

$$
\begin{array}{r}
\dot{V}\left(e_{y}, e_{z}, \tilde{\theta}\right)=2 \alpha a_{m} e_{y}^{2}-2 \alpha g\left(z_{m}-e_{z}\right) e_{y}+2 \beta h e_{z}^{2}+2 \beta\left(l+m k_{y}^{*}\right) e_{y} e_{z}-2 \beta m \tilde{k}_{y} y e_{z}+2 \alpha v b^{2} a_{m}^{-1} \tilde{k}_{y}\left(\tilde{k}_{y}+k_{y}^{*}\right) y^{2} \\
\leq-2 \alpha\left|a_{m}\right|\left\|e_{y}\right\|^{2}+2 \alpha|g|\left\|z_{m}\right\|\left\|e_{y}\right\|-2 \beta|h|\left\|e_{z}\right\|^{2}+2\left|\alpha g+\beta\left(l+m k_{y}^{*}\right)\right|\left\|e_{y}\right\|\left\|e_{z}\right\|+2 \beta|m|\|y\|\left\|\tilde{k}_{y}\right\|\left\|e_{z}\right\| \\
-2 \alpha v b^{2}\left|a_{m}^{-1}\right|\|y\|^{2}\left\|\tilde{k}_{y}\right\|^{2}+2 \alpha v b^{2}\left|a_{m}^{-1}\right|\left|k_{y}^{*}\right|\|y\|^{2}\left\|\tilde{k}_{y}\right\|
\end{array}
$$

Using the inequality $2\|a\|\|b\| \leq \delta^{2}\|a\|^{2}+\frac{\|b\|^{2}}{\delta^{2}}$, we get

$$
\begin{array}{r}
\dot{V}\left(e_{y}, e_{z}, \tilde{\theta}\right) \leq-2 \alpha\left|a_{m}\right|\left\|e_{y}\right\|^{2}+2 \alpha|g|\left\|z_{m}\right\|\left\|e_{y}\right\|-2 \beta|h|\left\|e_{z}\right\|^{2}+\left|\alpha g+\beta\left(l+m k_{y}^{*}\right)\right|\left(\delta_{1}^{2}\left\|e_{y}\right\|^{2}+\frac{\left\|e_{z}\right\|^{2}}{\delta_{1}^{2}}\right) \\
+\beta|m|\left(\delta_{2}^{2}\|y\|^{2}\left\|\tilde{k}_{y}\right\|^{2}+\frac{\left\|e_{z}\right\|^{2}}{\delta_{2}^{2}}\right)-2 \alpha v b^{2}\left|a_{m}^{-1}\right|\|y\|^{2}\left\|\tilde{k}_{y}\right\|^{2}+2 \alpha v b^{2}\left|a_{m}^{-1}\right|\left|k_{y}^{*}\right|\|y\|^{2}\left\|\tilde{k}_{y}\right\|
\end{array}
$$

Note that the negative definite term $-2 \alpha v b^{2}\left|a_{m}^{-1}\right|\|y\|^{2}\left\|\tilde{k}_{y}\right\|^{2}$ of the optimal control modification can be made to dominate the positive definite term $\beta|m| \delta_{2}^{2}\|y\|^{2}\left\|\tilde{k}_{y}\right\|^{2}$ to enable $\dot{V}(e, \tilde{\theta}, z)$ to be negative definite.

Let $c_{1}=2 \alpha\left|a_{m}\right|-\left|\alpha g+\beta\left(l+m k_{y}^{*}\right)\right| \delta_{1}^{2}, c_{2}=2 \beta|h|-\frac{\left|\alpha g+\beta\left(l+m k_{y}^{*}\right)\right|}{\delta_{1}^{2}}-\frac{\beta|m|}{\delta_{2}^{2}}, c_{3}=2 \alpha v b^{2}\left|a_{m}^{-1}\right|-\beta|m| \delta_{2}^{2}$, and $c_{4}=\frac{\alpha v b^{2}\left|a_{m}^{-1}\right|\left|k_{y}^{*}\right|}{c_{3}}$. Then,

$$
\dot{V}\left(e_{y}, e_{z}, \tilde{\theta}\right) \leq-c_{1}\left\|e_{y}\right\|^{2}+2 \alpha|g|\left\|z_{m}\right\|\left\|e_{y}\right\|-c_{2}\left\|e_{z}\right\|^{2}-c_{3}\|y\|^{2}\left(\left\|\tilde{k}_{y}\right\|-c_{4}\right)^{2}+c_{3} c_{4}^{2}\|y\|^{2}
$$

We note that $\|y\|^{2} \leq\left\|e_{y}\right\|^{2}+2\left\|e_{y}\right\|\left\|y_{m}\right\|+\left\|y_{m}\right\|^{2}$. The ultimate bounds of $\left\|y_{m}\right\|$ and $\left\|z_{m}\right\|$ can be shown to be $\left\|y_{m}\right\| \leq c_{y} r_{0}$ and $\left\|z_{m}\right\| \leq c_{z} r_{0}$ where $c_{y}=\left|a_{m}^{-1} b_{m}\right|, c_{z}=\left|h^{-1} m k_{r}\right|+\left|h^{-1}\left(l+m k_{y}^{*}\right)\right|\left|a_{m}^{-1} b_{m}\right|$, and $r_{0}=\|r\|$. Let $c_{5}=c_{1}-c_{3} c_{4}^{2}, c_{6}=\frac{\left(\alpha|g| c_{z}+c_{3} c_{4}^{2} c_{y}\right) r_{0}}{c_{5}}$, and $c_{7}=c_{5} c_{6}^{2}+c_{3} c_{4}^{2} c_{y}^{2} r_{0}^{2}$. Then,

$$
\dot{V}\left(e_{y}, e_{z}, \tilde{\theta}\right) \leq-c_{5}\left(\left\|e_{y}\right\|-c_{6}\right)^{2}-c_{2}\left\|e_{z}\right\|^{2}-c_{3}\|y\|^{2}\left(\left\|\tilde{k}_{y}\right\|-c_{4}\right)^{2}+c_{7}
$$

$v, \alpha, \beta, \delta_{1}$, and $\delta_{2}$ are chosen such that $c_{2}>0, c_{3}>0$, and $c_{5}>0$. Then, it follows that $\dot{V}\left(e_{y}, e_{z}, \tilde{\theta}\right) \leq 0$ if

$$
\begin{gathered}
\left\|e_{y}\right\| \geq c_{6}+\sqrt{\frac{c_{7}}{c_{5}}}=p \\
\left\|e_{z}\right\| \geq \sqrt{\frac{c_{7}}{c_{2}}}=q \\
\left\|\tilde{k}_{y}\right\| \geq c_{4}+\sqrt{\frac{c_{7}}{c_{3}\left(p+c_{y} r_{0}\right)^{2}}}=\kappa
\end{gathered}
$$

The ultimate bound of $\left\|e_{y}\right\|$ is then obtained as

$$
\left\|e_{y}\right\| \leq \sqrt{p^{2}+\frac{\beta}{\alpha} q^{2}+\gamma_{y}^{-1} \kappa^{2}}
$$

Thus, the closed-loop non-minimum phase system is stable with output feedback adaptive control with the optimal control modification. 
It should be noted that the aforementioned output feedback adaptive control design is feasible only if the knowledge of the plant transfer function is mostly known with the exception of the parameter $a$. This requirement imposes a severe restriction on the design of output feedback adaptive control for non-minimum phase plants since in many cases such knowledge may not exist if the plants are uncertain. Another drawback with this approach is that the desired tracking performance is not guaranteed. In practical situations, this poor tracking performance can be a major issue.

Example 1: Consider the system (2) with $a<0$ is unknown but $a=-2$ for simulation purposes, and $b=1$ is known. The reference model is given by the transfer function $W_{m}(s)$ with $a_{m}=-1$ and $b_{m}=1$.

The open-loop transfer function is

$$
W_{p}(s)=\frac{s+1+g}{(s-a)(s+1)}
$$

The system is minimum phase if $g>-1$. Consider a minimum phase plant with $g=2$. Then, we design the output feedback adaptive controller according to Eq. (18). Let $\lambda=-1, \Gamma=I$, and $r(t)=1$. Figure 1 shows the response of the closed-loop plant which tracks the reference model very well in the limit as $t \rightarrow \infty$.
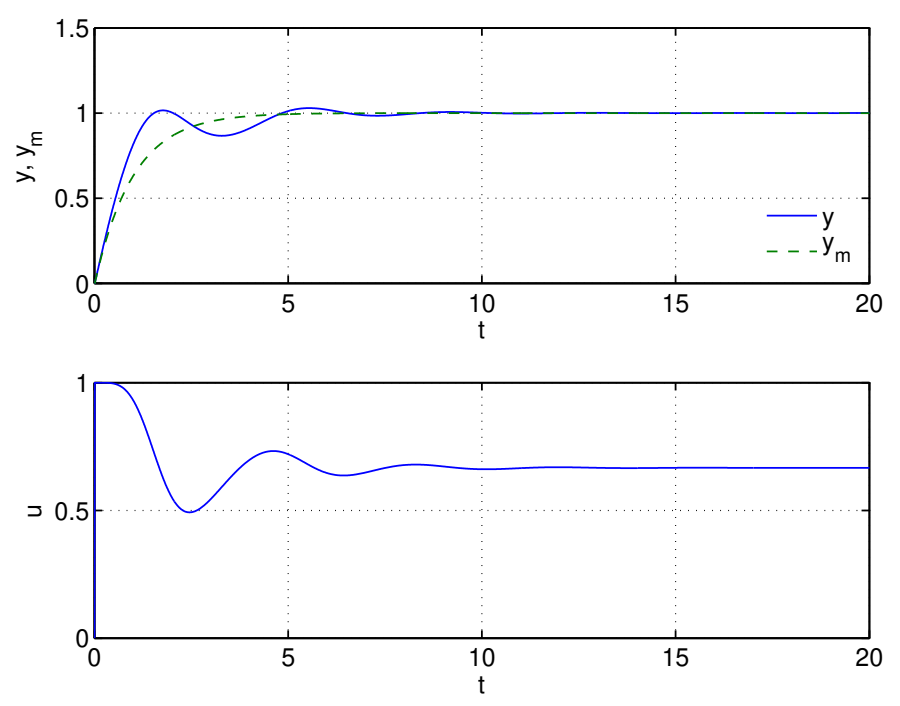

Figure 1: Closed-Loop Response of Minimum Phase Plant with MRAC

Consider a non-minimum phase plant with $g=-2$. We use both adaptive controllers given by Eq. (18) and (32). The asymptotic closed-loop transfer function is given by

$$
\frac{y}{r}=\frac{(s-1)\left[v b_{m}\left(s-a_{m}\right)-a_{m} b_{m}\right]}{\left(s-a_{m}\right)\left[v(s-a)(s+1)-a_{m}(s-1)\right]}
$$

The steady-state closed-loop transfer function is equal to

$$
\frac{\bar{y}}{r}=\frac{b_{m}(v+1)}{v a-a_{m}}
$$

For $a=-2$ and $a_{m}=-1$, the transfer function is stable for $v>0.5$. Choose $v=2$ and $\gamma_{y}=1$. The steadystate closed-loop output is equal to $\bar{y}(t)=-1$. Figure 2 shows the stable closed-loop responses of the non-minimum phase plant with both the adaptive controllers. The adaptive controller 1 with the adaptive parameter $\Theta(t)$ has a faster response than the adaptive controller 2 with the adaptive parameter $k_{y}(t)$. Both adaptive controllers tend to the same steady-state closed-loop response.The closed-loop plant tracks the reference model very poorly as expected, but the 
response is stable and tends to an equilibrium value $\bar{y}=-1$ which agrees with the analytical result from the steadystate closed-loop transfer function. The poor tracking performance is typical of a non-minimum phase system with the output generally exhibiting an opposite response to the input.
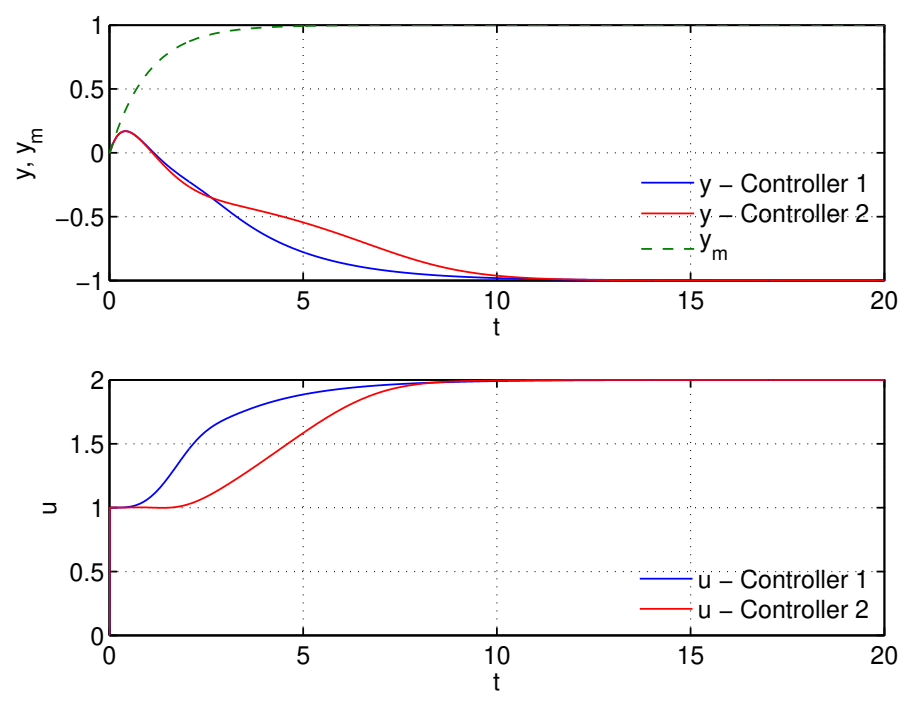

Figure 2: Closed-Loop Response of Non-Minimum Phase Plant with Optimal Control Modification

\section{Output Feedback Adaptive Control of Non-SPR MIMO Systems with Uni- form Relative Degree 1}

Given the following MIMO system

$$
\begin{aligned}
\dot{x}=A x+B u & \Leftrightarrow\left[\begin{array}{c}
\dot{x}_{1} \\
\dot{x}_{2}
\end{array}\right]=\left[\begin{array}{ll}
A_{11} & A_{12} \\
A_{21} & A_{22}
\end{array}\right]\left[\begin{array}{l}
x_{1} \\
x_{2}
\end{array}\right]+\left[\begin{array}{l}
B_{1} \\
B_{2}
\end{array}\right] u \\
y & =C x=\left[\begin{array}{ll}
C_{1} & 0
\end{array}\right]\left[\begin{array}{l}
x_{1} \\
x_{2}
\end{array}\right]=C_{1} x_{1}
\end{aligned}
$$

where $x_{1}(t) \in \mathbb{R}^{p}, x_{2}(t) \in \mathbb{R}^{n-p}, u(t) \in \mathbb{R}^{m}, y(t) \in \mathbb{R}^{p}$ with $p \leq m, A_{11} \in \mathbb{R}^{p \times p}$ is unknown, $A_{12} \in \mathbb{R}^{p \times(n-p)}$ and $A_{21} \in \mathbb{R}^{(n-p) \times p}$ are known, $A_{22} \in \mathbb{R}^{(n-p) \times(n-p)} \in \mathbb{C}^{-}$is known and assumed to be Hurwitz, $B_{1} \in \mathbb{R}^{p \times m}$ is known and has full rank, $B_{2} \in \mathbb{R}^{(n-p) \times m}$ is known, and $C_{1} \in \mathbb{R}^{p \times p}$ is known and has full rank. We assume the pair $(A, B)$ is controllable and the pair $(A, C)$ is observable. This partitioned form of the plant can be used advantageously to design an output feedback adaptive control. The system expressed in this form is sufficiently general for many practical applications. For example, systems with unmodeled dynamics could be expressed in this form with $x_{1}(t)$ being the plant state variable and $x_{2}(t)$ being the unmodeled state variable.

Expressed in this form, the MIMO system (50) has a uniform relative degree 1 since $u(t)$ appears in the first derivative of $y(t)$

$$
\dot{y}=C_{1} \dot{x}_{1}=C_{1} A_{11} C_{1}^{-1} y+C_{1} A_{12} x_{2}+C_{1} B_{1} u
$$

and $\operatorname{rank}\left(C_{1} B_{1}\right)=p$ which implies that $C_{1} B_{1}$ is invertible. The condition of invertibility of $C_{1} B_{1}$ allows the design of a model-following output feedback controller for the output $y(t)$ to track a reference model of the same relative degree.

The Kalman-Yakubovich lemma [8] can be used to determine if the transfer function matrix $G(s)=C(s I-A)^{-1} B$ is SPR. If $A$ is Hurwitz and there exist $P=P^{\top} \in \mathbb{R}^{n \times n}>0$ and $Q=Q^{\top}>0 \in \mathbb{R}^{r \times n}$ such that

$$
P A+A^{\top} P=-Q
$$




$$
B^{\top} P=C
$$

then $G(s)$ is SPR. Equation (53) leads to the following necessary and sufficient condition for a SPR transfer function matrix [9]:

$$
B^{\top} P B=C B>0
$$

The condition $C B>0$ requires $C B$ to be a symmetric positive definite square matrix. $G(s)$ is non-SPR for this system if $p<m$ and SPR if $p=m$ and $C B=C_{1} B_{1}>0$.

The objective is to design an output feedback to track the following reference model:

$$
\dot{y}_{m}=A_{m} y_{m}+B_{m} r
$$

where $A_{m} \in \mathbb{R}^{p \times p}$ is Hurwitz and $r(t) \in \mathbb{R}^{q} \in \mathscr{L}_{\infty}$ with $q \leq m$ is a bounded command signal. Furthermore, the transfer function matrix $G_{m}(s)=\left(s I-A_{m}\right)^{-1} B_{m}$ is assumed to be SPR. If $G(s)$ is non-SPR and $G_{m}(s)$ is SPR, there is no guarantee of a stable adaptation for output feedback adaptive control. However, under a certain condition, a stable adaptation for output feedback adaptive control is possible.

Toward establishing this condition, the dynamics of the output can be expressed as

$$
\dot{y}=C_{1} A_{11} C_{1}^{-1} y+C_{1} A_{12} x_{2}+C_{1} B_{1} u
$$

Let $x_{2}(t)=\Phi_{y}(t)+\Phi_{u}(t)$ where $\Phi_{y}(t) \in \mathbb{R}^{n-p}$ and $\Phi_{u}(t) \in \mathbb{R}^{n-p}$ have the following dynamics

$$
\begin{gathered}
\dot{\Phi}_{y}=A_{22} \Phi_{y}+A_{21} C_{1}^{-1} y \\
\dot{\Phi}_{u}=A_{22} \Phi_{u}+B_{2} u
\end{gathered}
$$

The ideal output feedback controller can be computed as

$$
u^{*}=\left(C_{1} B_{1}\right)^{+}\left[B_{m} r+\left(A_{m}-C_{1} A_{11} C_{1}^{-1}\right) y-C_{1} A_{12}\left(\Phi_{y}+\Phi_{u}\right)\right]
$$

where $\left(C_{1} B_{1}\right)^{+}=\left(C_{1} B_{1}\right)^{\top}\left[\left(C_{1} B_{1}\right)\left(C_{1} B_{1}\right)^{\top}\right]^{-1}$ is the pseudo inverse of $C_{1} B_{1}$ which is non-singular for $p \leq m$. This is effectively an optimal control allocation strategy when the number of inputs exceeds the number of outputs.

The adaptive controller is then given by

$$
u=-\left(C_{1} B_{1}\right)^{+}\left(\Theta_{y}^{\top} y+\Theta_{\Phi_{y}}^{\top} \Phi_{y}+\Theta_{\Phi_{u}}^{\top} \Phi_{u}+\Theta_{r}^{\top} r\right)=-\left(C_{1} B_{1}\right)^{+} \Theta^{\top} \Phi(y, u, r)
$$

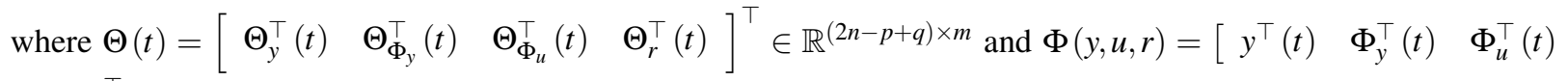
$\left.r^{\top}(t)\right]^{\top} \in \mathbb{R}^{2 n-p+q}$. Let $\Theta^{*}$ be the ideal unknown matrix. Then, the model matching condition results in $\Theta_{y}^{*}=$ $-\left(A_{m}-C_{1} A_{11} C_{1}^{-1}\right)^{\top}, \Theta_{\Phi_{y}}^{*}=\left(C_{1} A_{12}\right)^{\top}, \Theta_{\Phi_{u}}^{*}=\left(C_{1} A_{12}\right)^{\top}$, and $\Theta_{r}^{*}=-B_{m}^{\top}$.

The output tracking error is defined as $e_{y}(t)=y_{m}(t)-y(t)$. Then,

$$
\dot{e}_{y}=A_{m} e_{y}+\tilde{\Theta}^{\top} \Phi(y, u)
$$

The output feedback adaptive law for the standard MRAC is given by

$$
\dot{\Theta}=-\Gamma \Phi(y, u, r) e_{y}^{\top} P
$$

where $P=P^{\top}>0$ solve the Lyapunov equation

$$
P A_{m}+A_{m}^{\top} P=-Q
$$

and $Q=Q^{\top}>0$.

We now state the following theorem for a stable adaptation for the standard MRAC.

Theorem 3: Let $F_{1}^{*}=-B_{2}\left(C_{1} B_{1}\right)^{+} \Theta_{y}^{* \top}, \quad F_{2}^{*}=-B_{2}\left(C_{1} B_{1}\right)^{+} \Theta_{\Phi_{y}}^{* \top}, \quad F_{3}^{*}=A_{22}-B_{2}\left(C_{1} B_{1}\right)^{+} \Theta_{\Phi_{u}}^{* \top}$, and $F_{4}^{*}=-B_{2}\left(C_{1} B_{1}\right)^{+} \Theta_{r}^{* \top}$, then the MIMO system (50) with the ideal controller is stable if $\lambda\left(F_{3}^{*}\right) \in \mathbb{C}^{-}$, that is, $F_{3}^{*}$ is Hurwitz. 
Proof: The combined dynamics of the output feedback control system due to the ideal controller are obtained as

$$
\left[\begin{array}{c}
\dot{y}_{m} \\
\dot{\Phi}_{y}^{*} \\
\dot{\Phi}_{u}^{*}
\end{array}\right]=\underbrace{\left[\begin{array}{ccc}
A_{m} & 0 & 0 \\
A_{21} C_{1}^{-1} & A_{22} & 0 \\
F_{1}^{*} & F_{2}^{*} & F_{3}^{*}
\end{array}\right]}_{F^{*}}\left[\begin{array}{c}
y_{m} \\
\Phi_{y}^{*} \\
\Phi_{u}^{*}
\end{array}\right]+\left[\begin{array}{c}
B_{m} \\
0 \\
F_{4}^{*}
\end{array}\right] r
$$

The eigenvalues of $F^{*}$ are computed from the following determinant formula:

$$
\begin{array}{r}
\operatorname{det}\left(F^{*}-s I\right)=\operatorname{det}\left(\left[\begin{array}{cc}
A_{m}-s I & 0 \\
A_{21} C_{1}^{-1} & A_{22}-s I
\end{array}\right]\right) \operatorname{det}\left(F_{3}^{*}-s I-\left[\begin{array}{cc}
F_{1}^{*} & F_{2}^{*}
\end{array}\right]\left[\begin{array}{cc}
A_{m}-s I & 0 \\
A_{21} C_{1}^{-1} & A_{22}-s I
\end{array}\right]^{-1}\left[\begin{array}{l}
0 \\
0
\end{array}\right]\right) \\
=\operatorname{det}\left(A_{m}-s I\right) \operatorname{det}\left(A_{22}-s I\right) \operatorname{det}\left(F_{3}^{*}-s I\right)
\end{array}
$$

Since $A_{m}$ and $A_{22}$ are Hurwitz, then $F^{*}$ is Hurwitz if $\lambda\left(F_{3}^{*}\right) \in \mathbb{C}^{-}$. Therefore, the output feedback control system is stable if $\Phi(y, u, r)$ is bounded which requires $\lambda\left(F_{3}^{*}\right) \in \mathbb{C}^{-}$.

Let $z(t)=\left[\begin{array}{ll}\Phi_{y}^{\top}(t) & \Phi_{u}^{\top}(t)\end{array}\right]^{\top} \in \mathbb{R}^{2 n-2 p}$ and define $e_{z}(t)=z^{*}(t)-z(t)$. Then,

$$
\dot{e}_{z}=F_{22}^{*} e_{z}+F_{21}^{*} e_{y}+E \tilde{\Theta}^{\top} \Phi(y, u, r)
$$

where $F_{21}^{*}=\left[\begin{array}{c}A_{21} C_{1}^{-1} \\ F_{1}^{*}\end{array}\right], F_{22}^{*}=\left[\begin{array}{cc}A_{22} & 0 \\ F_{2}^{*} & F_{3}^{*}\end{array}\right]$, and $E=\left[\begin{array}{c}0 \\ B_{2}\left(C_{1} B_{1}\right)^{+}\end{array}\right]$.

Suppose $\Phi(y, u, r)$ is bounded which requires $F_{3}^{*}$ to be Hurwitz and implies that $e_{z}(t)$ is also bounded. Then, we choose the Lyapunov candidate function

$$
V\left(e_{y}, \tilde{\Theta}\right)=e_{y}^{\top} P e_{y}+\operatorname{trace}\left(\tilde{\Theta}^{\top} \Gamma^{-1} \tilde{\Theta}\right)
$$

Then,

$$
\dot{V}\left(e_{y}, \tilde{\Theta}\right)=-e_{y}^{\top} Q e_{y} \leq-\lambda_{\min }(Q)\left\|e_{y}\right\|^{2} \leq 0
$$

Therefore, $e_{y}(t) \in \mathscr{L}_{2} \cap \mathscr{L}_{\infty}$ and $\tilde{\Theta}(t) \in \mathscr{L}_{\infty}$ are bounded. This implies Eq. (66) admits a bounded solution of $e_{z}(t)$ which validates the supposition of boundedness of $\Phi(y, u, r)$. Note that $\ddot{V}\left(e_{y}, \tilde{\Theta}\right)$ is bounded since $\Phi(y, u, r)$ is bounded. Therefore, $\dot{V}\left(e_{y}, \tilde{\Theta}\right)$ is uniformly continuous. Invoking the Barbalat's lemma, it follows that the output tracking error tends to zero asymptotically with $e_{y}(t) \rightarrow 0$ as $t \rightarrow \infty$. This also implies $\tilde{\Theta}^{\top}(t) \Phi(y, u, r) \rightarrow 0$ as $e_{y}(t) \rightarrow 0$. Therefore, $e_{z}(t) \rightarrow 0$ as $t \rightarrow \infty$ if $\lambda\left(F_{3}^{*}\right) \in \mathbb{C}^{-}$. It is obvious that, if $\lambda\left(F_{3}^{*}\right) \notin \mathbb{C}^{-}$, then $\Phi(y, u, r)$ is unbounded and the standard MRAC output feedback adaptive law is unstable.

To achieve a stable adaptation for the non-SPR plant (50) with $\lambda\left(F_{3}^{*}\right) \notin \mathbb{C}^{-}$, the optimal control modification to the adaptive law in Eq. (61) is proposed as

$$
\dot{\Theta}=-\Gamma \Phi(y, u, r)\left[e_{y}^{\top} P-v \Phi^{\top}(y, u, r) \Theta P A_{m}^{-1}\right]
$$

where $v>0$.

We examine the linear asymptotic property of the optimal control modification adaptive law as $\Gamma \rightarrow \infty$. Then, in the limit, one gets

$$
\bar{\Theta}^{\top} \Phi(y, u, r)=\frac{1}{v} P^{-1} A_{m}^{\top} P e_{y}
$$

where the bar symbol denotes the value in the limit.

The closed-loop system in the limit is now defined by

$$
\begin{array}{r}
{\left[\begin{array}{c}
\dot{\bar{y}} \\
\dot{\bar{\Phi}}_{y} \\
\dot{\bar{\Phi}}_{u}
\end{array}\right]=\underbrace{\left[\begin{array}{ccc}
C_{1} A_{11} C_{1}^{-1}+\frac{1}{v} P^{-1} A_{m}^{\top} P & C_{1} A_{12} & C_{1} A_{12} \\
A_{21} C_{1}^{-1} & A_{22} & 0 \\
\frac{1}{v} B_{2}\left(C_{1} B_{1}\right)^{+} P^{-1} A_{m}^{\top} P & 0 & A_{22}
\end{array}\right]}_{G}\left[\begin{array}{c}
\bar{y} \\
\bar{\Phi}_{y} \\
\bar{\Phi}_{u}
\end{array}\right]+\left[\begin{array}{c}
B_{m} \\
0 \\
B_{2}\left(C_{1} B_{1}\right)^{+} B_{m}
\end{array}\right] r} \\
-\frac{1}{v}\left[\begin{array}{c}
P^{-1} A_{m}^{\top} P \\
0 \\
B_{2}\left(C_{1} B_{1}\right)^{+} P^{-1} A_{m}^{\top} P
\end{array}\right] y_{m}
\end{array}
$$


Then, a stable adaptation can be achieved with the optimal control modification by a suitable selection of $v$ such that $\lambda(G) \in \mathbb{C}^{-}$. We express the error equation for $z(t)$ as

$$
\dot{e}_{z}=A_{z z} e_{z}+A_{z y} e_{y}+E \tilde{\Theta}^{\top} \Phi(y, u, r)
$$

where $A_{z z}=\left[\begin{array}{cc}A_{22} & 0 \\ 0 & A_{22}\end{array}\right]$ and $A_{z y}=\left[\begin{array}{c}A_{21} C_{1}^{-1} \\ 0\end{array}\right]$.

Theorem 4: The optimal control modification adaptive law is stable for the non-SPR plant (50) with $\lambda\left(F_{3}^{*}\right) \notin \mathbb{C}^{-}$ by a suitable selection of the modification $v$ such that $\|\Phi(y, u, r)\| \leq \Phi_{0} \in \mathscr{L}_{\infty}$. Then, $e_{y}(t)$ is uniformly ultimately bounded.

Proof: Choose a Lyapunov candidate function

$$
V\left(e_{y}, e_{z}, \tilde{\Theta}\right)=e_{y}^{\top} P e_{y}+e_{z}^{\top} W e_{z}+\operatorname{trace}\left(\tilde{\Theta}^{\top} \Gamma^{-1} \tilde{\Theta}\right)
$$

where $W=W^{\top}>0$ that solves the Lyapunov equation

$$
W A_{z z}+A_{z z}^{\top} W=-R
$$

with $R=R^{\top}>0$.

Then,

$\dot{V}\left(e_{y}, e_{z}, \tilde{\Theta}\right)=-e_{y}^{\top} Q e_{y}-e_{z}^{\top} R e_{z}+2 e_{z}^{\top} W A_{z y} e_{y}+2 e_{z}^{\top} W E \tilde{\Theta}^{\top} \Phi(y, u, r)+2 v \Phi^{\top}(y, u, r) \Theta P A_{m}^{-1} \tilde{\Theta}^{\top} \Phi(y, u, r)$

Let $c_{1}=\lambda_{\min }(Q), c_{2}=\lambda_{\min }(R), c_{3}=\left\|W A_{z y}\right\|, c_{4}=\|W E\|, c_{5}=v \lambda_{\min }\left(A_{m}^{-\top} Q A_{m}^{-1}\right)$, and $c_{6}=v\left\|P A_{m}^{-1}\right\|\left\|\Theta^{*}\right\|$. Then, $\dot{V}\left(e_{y}, e_{z}, \tilde{\Theta}\right)$ is bounded by

$$
\begin{array}{r}
\dot{V}\left(e_{y}, e_{z}, \tilde{\Theta}\right) \leq-\left(c_{1}-\frac{c_{3}}{\delta^{2}}\right)\left\|e_{y}\right\|^{2}-\left(c_{2}-c_{3} \delta^{2}-c_{4} \varepsilon^{2}\right)\left\|e_{z}\right\|^{2}-\left(c_{5}-\frac{c_{4}}{\varepsilon^{2}}\right)\|\Phi(y, u, r)\|^{2}\|\tilde{\Theta}\|^{2} \\
+2 c_{6}\|\Phi(y, u, r)\|^{2}\|\tilde{\Theta}\|
\end{array}
$$

Note that $\|\Phi(y, u, r)\|^{2} \leq\|y\|^{2}+\|z\|^{2}+r_{0}^{2} \leq\left\|e_{y}\right\|^{2}+2 c_{y} r_{0}\left\|e_{y}\right\|+\left\|e_{z}\right\|^{2}+2 c_{z} r_{0}\left\|e_{z}\right\|+\left(1+c_{y}^{2}+c_{z}^{2}\right) r_{0}^{2}$. Then,

$$
\begin{aligned}
\dot{V}\left(e_{y}, e_{z}, \tilde{\Theta}\right) \leq-\left(c_{1}-\right. & \left.\frac{c_{3}}{\delta^{2}}-\frac{c_{6}^{2}}{c_{5}-\frac{c_{4}}{\varepsilon^{2}}}\right)\left\|e_{y}\right\|^{2}+\frac{2 c_{6}^{2} c_{y} r_{0}}{c_{5}-\frac{c_{4}}{\varepsilon^{2}}}\left\|e_{y}\right\|-\left(c_{2}-c_{3} \delta^{2}-c_{4} \varepsilon^{2}-\frac{c_{6}^{2}}{c_{5}-\frac{c_{4}}{\varepsilon^{2}}}\right)\left\|e_{z}\right\|^{2} \\
& +\frac{2 c_{6}^{2} c_{z} r_{0}}{c_{5}-\frac{c_{4}}{\varepsilon^{2}}}\left\|e_{z}\right\|-\left(c_{5}-\frac{c_{4}}{\varepsilon^{2}}\right)\|\Phi(y, u, r)\|^{2}\left(\|\tilde{\Theta}\|-\frac{c_{6}}{c_{5}-\frac{c_{4}}{\varepsilon^{2}}}\right)^{2}+\frac{c_{6}^{2}\left(1+c_{y}^{2}+c_{z}^{2}\right) r_{0}^{2}}{c_{5}-\frac{c_{4}}{\varepsilon^{2}}}
\end{aligned}
$$

Let $c_{7}=c_{1}-\frac{c_{3}}{\delta^{2}}-\frac{c_{6}^{2}}{c_{5}-\frac{c_{4}}{\varepsilon^{2}}}, c_{8}=\frac{c_{6}^{2} c_{y} r_{0}}{c_{7}\left(c_{5}-\frac{c_{4}}{\varepsilon^{2}}\right)}, c_{9}=c_{2}-c_{3} \delta^{2}-c_{4} \varepsilon^{2}-\frac{c_{6}^{2}}{c_{5}-\frac{c_{4}}{\varepsilon^{2}}}, c_{10}=\frac{c_{6}^{2} c_{2} r_{0}}{c_{9}\left(c_{5}-\frac{c_{4}}{\varepsilon^{2}}\right)}, c_{11}=c_{5}-\frac{c_{4}}{\varepsilon^{2}}, c_{12}=$ $\frac{c_{6}}{c_{5}-\frac{c_{4}}{\varepsilon^{2}}}$, and $c_{13}=\frac{c_{6}^{2}\left(1+c_{y}^{2}+c_{z}^{2}\right) r_{0}^{2}}{c_{5}-\frac{c_{4}}{\varepsilon^{2}}}$. Choose $Q, R, v, \delta$, and $\varepsilon$ such that $c_{7}>0, c_{9}>0$, and $c_{11}>0$. Then, $\dot{V}\left(e_{y}, e_{z}, \tilde{\Theta}\right) \leq 0$ outside the compact set

$$
\begin{aligned}
\mathscr{S}=\left\{e_{y}(t) \in \mathbb{R}^{p}, e_{z}(t) \in \mathbb{R}^{2 n-2 p}, \tilde{\Theta}(t) \in \mathbb{R}^{(2 n-p+q) \times m}: c_{7}\left(\left\|e_{y}\right\|-c_{8}\right)^{2}+c_{9}\left(\left\|e_{z}\right\|-c_{10}\right)^{2}\right. \\
\left.+c_{11}\|\Phi(y, u, r)\|^{2}\left(\|\tilde{\Theta}\|-c_{12}\right)^{2} \leq c_{7} c_{8}^{2}+c_{9} c_{10}^{2}+c_{13}\right\}
\end{aligned}
$$

if

$$
\left\|e_{y}\right\| \geq c_{8}+\sqrt{\frac{c_{7} c_{8}^{2}+c_{9} c_{10}^{2}+c_{13}}{c_{7}}}=p
$$




$$
\begin{aligned}
& \left\|e_{z}\right\| \geq c_{10}+\sqrt{\frac{c_{7} c_{8}^{2}+c_{9} c_{10}^{2}+c_{13}}{c_{9}}}=q \\
& \|\tilde{\Theta}\| \geq c_{12}+\sqrt{\frac{c_{7} c_{8}^{2}+c_{9} c_{10}^{2}+c_{13}}{c_{11} \Phi_{0}^{2}}}=\kappa
\end{aligned}
$$

where $\Phi_{0}^{2}=\left(p+c_{y} r_{0}\right)^{2}+\left(q+c_{z} r_{0}\right)^{2}+r_{0}^{2}$.

Therefore, the optimal control modification adaptive law is stable and all signals are uniformly ultimately bounded. The ultimate bound of the output tracking error is determined to be

$$
\left\|e_{y}\right\| \leq \sqrt{\frac{\lambda_{\max }(P) p^{2}+\lambda_{\max }(W) q^{2}+\lambda_{\max }\left(\Gamma^{-1}\right) \kappa^{2}}{\lambda_{\min }(P)}}
$$

Example 2: Given

$$
\begin{aligned}
A=\left[\begin{array}{cccc}
0 & 1 & -1 & 0 \\
-1 & -4 & 0 & g \\
0 & 0 & 0 & 1 \\
0 & 0 & -5 & -10
\end{array}\right], B=\left[\begin{array}{ccc}
0 & 1 & 0 \\
1 & 2 & -1 \\
2 & 0 & 0 \\
3 & 4 & 0
\end{array}\right], C=\left[\begin{array}{llll}
1 & 2 & 0 & 0 \\
2 & 1 & 0 & 0
\end{array}\right], D=\left[\begin{array}{lll}
0 & 0 & 0 \\
0 & 0 & 0
\end{array}\right] \\
A_{m}=\left[\begin{array}{cc}
0 & 1 \\
-2 & -4
\end{array}\right], B_{m}=\left[\begin{array}{l}
0 \\
2
\end{array}\right]
\end{aligned}
$$

If $g=-1$, then $\lambda\left(F_{3}^{*}\right)=-3.6492,-6.8508$. Then, the standard MRAC update law with $\Gamma=1.01 \mathrm{I}$ is used for the output feedback adaptive controller. The response of the closed-loop plant to a unit step command is stable as shown in Fig. 3. The output $y(t)$ asymptotically tracks the reference model $y_{m}(t)$.
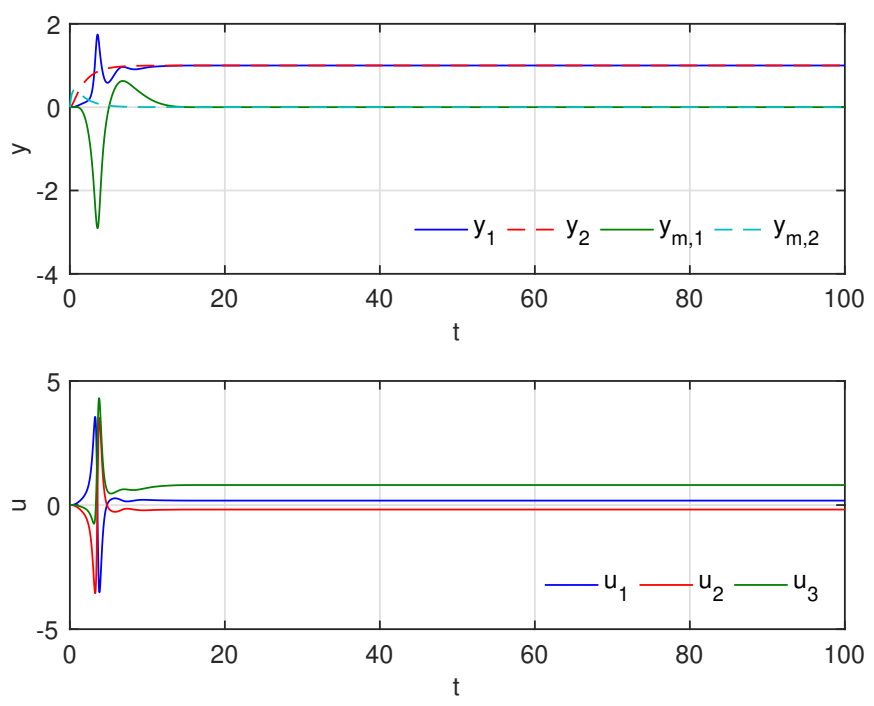

Figure 3: Closed-Loop Response of Non-SPR Plant $\lambda\left(F_{3}^{*}\right) \in \mathbb{C}^{-}$with Output Feedback MRAC

If $g=-10$, then $\lambda\left(F_{3}^{*}\right)=1.5 \pm 5.6347 i$. The standard MRAC update law results in unbounded signals. Figure 4 shows the response of the closed-loop plant with the standard MRAC update law which is oscillatory but eventually diverges as $t$ increases. The optimal control modification adaptive law, on the other hand, is able to stabilize the non-SPR plant with $v=0.1$ as shown in Fig. 5 . 

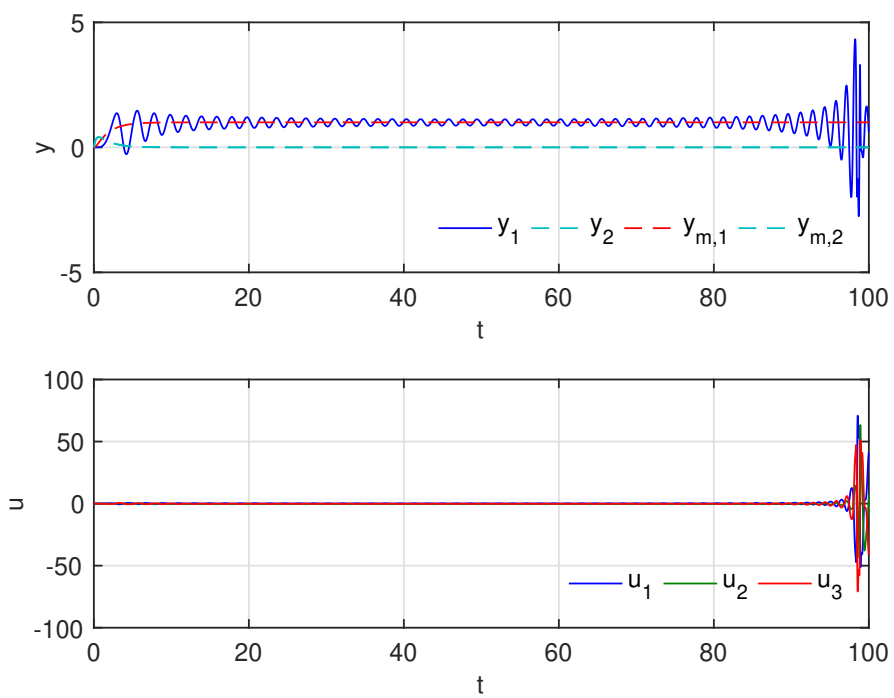

Figure 4: Closed-Loop Response of Non-SPR Plant $\lambda\left(F_{3}^{*}\right) \notin \mathbb{C}^{-}$with Output Feedback MRAC
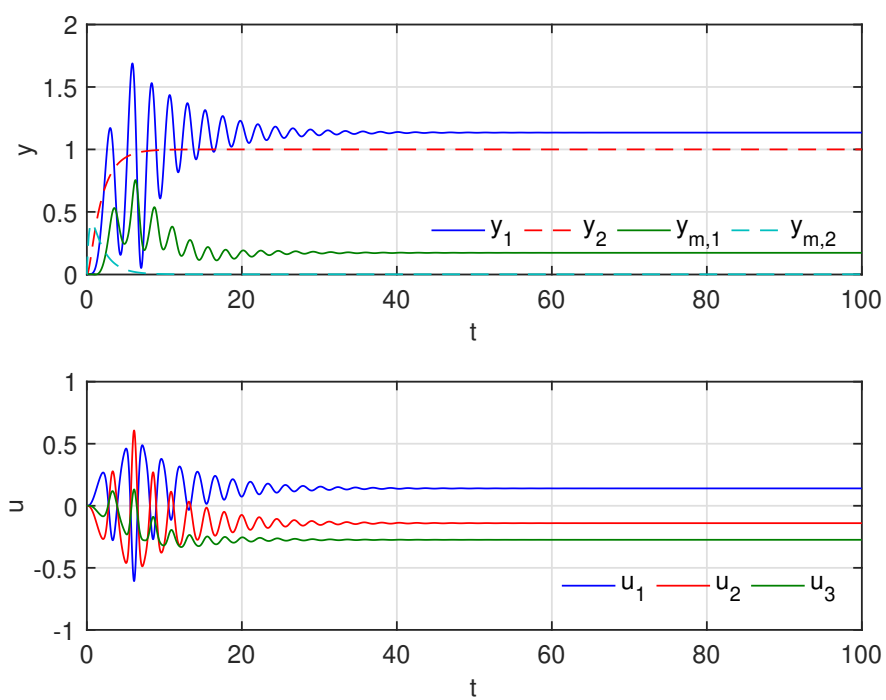

Figure 5: Closed-Loop Response of Non-SPR Plant $\lambda\left(F_{3}^{*}\right) \notin \mathbb{C}^{-}$with Optimal Control Modification

\section{Observer-Based Output Feedback Adaptive Control}

As can be seen from the Example 1, while the optimal control modification can stabilize a non-minimum phase plant, the tracking performance is quite unacceptable. The issue at hand is the requirement for a non-minimum phase plant to track a minimum phase reference model with the same relative degree. This is a very demanding and perhaps unrealistic requirement. As a consequence, MRAC attempts to seek asymptotic tracking which results in an unstable 
pole-zero cancellation. If the reference model could be redesigned so that the unstable pole-zero cancellation cannot occur while the tracking performance can still be met, then the output feedback adaptive control design would be more acceptable. One such approach is to design an observer state feedback adaptive control using the Luenberger observer design.

Consider the same MIMO system in Section 3

$$
\begin{gathered}
\dot{x}=A x+B u \\
y=C x
\end{gathered}
$$

where $x(t) \in \mathbb{R}^{n}, u(t) \in \mathbb{R}^{m}, y(t) \in \mathbb{R}^{p}$ with $p>m, A \in \mathbb{R}^{n} \times \mathbb{R}^{n}$ is Hurwitz but unknown, $B \in \mathbb{R}^{n} \times \mathbb{R}^{m}$ is known with the pair $(A, B)$ controllable, and $C \in \mathbb{R}^{p} \times \mathbb{R}^{n}$ is known with the pair $(A, C)$ observable. We assume that $A=A_{0}+\Delta A$ where $A_{0}$ is known and $\Delta A$ is an unknown perturbation of $\bar{A}$. The transfer function $G(s)=C(s I-A)^{-1} B$ is assumed to be non-SPR phase.

The Luenberger observer design constructs an observer state-space model of the plant as

$$
\dot{\hat{x}}=\hat{A} \hat{x}+L(y-\hat{y})+B u
$$

where $\hat{x}(t)$ is the observer state which estimates the plant state $x(t), \hat{A}(t)$ is the estimate of $A, \hat{y}(t)=C \hat{x}(t)$ is the observer output which estimates the plant output $y(t)$, and $L$ is the Kalman filter gain matrix computed using $A_{0}$.

A full-state feedback controller can be designed to enable the output $y(t)$ to track a reference command signal $r(t)$. For example, we can use the LQR method to design the full-state feedback controller using the following cost function for tracking a constant reference command signal $r(t)$ :

$$
J=\lim _{t_{f} \rightarrow \infty} \frac{1}{2} \int_{0}^{t_{f}}\left[(C x-r)^{\top} Q(C x-r)+u^{\top} R u\right] d t
$$

Then, the control gains can be computed as

$$
\begin{gathered}
K_{x}^{*}=-R^{-1} B^{\top} W \\
K_{r}=-R^{-1} B^{\top}\left(A^{\top}-W B R^{-1} B^{\top}\right)^{-1} C^{\top} Q
\end{gathered}
$$

where $W$ is the solution of the Riccati equation

$$
W A+A^{\top} W-W B R^{-1} B^{\top} W+C^{\top} Q C=0
$$

Then, a reference model is constructed from this LQR design with $A_{m}=A+B K_{x}^{*}$ and $B_{m}=B K_{r}$.

If $A$ is unknown, then we can design the following adaptive controller:

$$
u=K_{x}(t) \hat{x}+K_{r} r
$$

where the observer state $\hat{x}(t)$ replaces the plant state.

The tracking error is defined as $e(t)=x_{m}(t)-\hat{x}(t)$. We also define the state estimation error as $e_{p}(t)=x(t)-\hat{x}(t)$. Then, the error equations are

$$
\begin{gathered}
\dot{e}=A_{m} e-L C e_{p}-\tilde{A} \hat{x}-B \tilde{K}_{x} \hat{x} \\
\dot{e}_{p}=\left(A_{p}+\Delta A\right) e_{p}-\tilde{A} \hat{x}
\end{gathered}
$$

where $\tilde{A}(t)=\hat{A}(t)-A, \tilde{K}_{x}(t)=K_{x}(t)-K_{x}^{*}, A_{p}=A_{0}-L C$, and $A_{p}+\Delta A$ is Hurwitz by a suitable choice of $L$.

Note that the state estimation error signal $e_{p}(t)$ is generally not available, but for the class of MIMO systems under consideration, it can be constructed. Since $C_{1}$ is invertible, then $x_{1}(t)$ can be constructed from $y(t)$. Let $z(t)=x_{2}(t)$ be the internal state, then $z(t)$ can be computed from the following equation:

$$
\dot{z}=A_{22} z+A_{21} C_{1}^{-1} y+B_{2} u
$$

with $z(0)=z_{0}$ and $A_{21}, A_{22}$, and $B_{2}$ known.

Then, the plant state can be constructed as $x(t)=\left[\begin{array}{ll}C_{1}^{-1} y(t) & z(t)\end{array}\right]$. 
The stability of the observer output feedback adaptive control using the optimal control modification is now provided by the following theorem:

Theorem 5: A stable adaptation of the non-SPR plant (83) can be achieved by the optimal control modification adaptive laws based on the observer state $\hat{x}(t)$ and the constructed state $x(t)$ from the output $y(t)$ and the internal state $z(t)$

$$
\begin{gathered}
\dot{K}_{x}^{\top}=\Gamma_{x} \hat{x}\left(e^{\top} P+v \hat{x}^{\top} K_{x}^{\top} B^{\top} P A_{m}^{-1}\right) B \\
\dot{\hat{A}}^{\top}=\Gamma_{A} \hat{x}\left(e^{\top} P+e_{p}^{\top} W+\eta \hat{x}^{\top} \hat{A}^{\top} P A_{m}^{-1}\right)
\end{gathered}
$$

where $P=P^{\top}>0$ and $W=W^{\top}>0$ are solutions to the Lyapunov equations

$$
\begin{gathered}
P A_{m}+A_{m}^{\top} P=-Q \\
W A_{p}+A_{p}^{\top} W=-R
\end{gathered}
$$

with $Q=Q^{\top}>0$ and $R=R^{\top}>0$.

Proof: Choose a Lyapunov candidate function

$$
V\left(e, e_{p}, \tilde{K}_{x}, \tilde{A}\right)=e^{\top} P e+e_{p}^{\top} W e_{p}+\operatorname{trace}\left(\tilde{K}_{x} \Gamma_{x}^{-1} \tilde{K}_{x}^{\top}\right)+\operatorname{trace}\left(\tilde{A} \Gamma_{A}^{-1} \tilde{A}^{\top}\right)
$$

Then, $\dot{V}\left(e, e_{p}, \tilde{K}_{x}, \Delta \tilde{A}\right)$ is evaluated as

$$
\begin{aligned}
\dot{V}\left(e, e_{p}, \tilde{K}_{x}, \tilde{A}\right)= & -e^{\top} Q e-e_{p}^{\top} \bar{R} e_{p}-2 e^{\top} P L C e_{p}+2 v \hat{x}^{\top} K_{x}^{\top} B^{\top} P A_{m}^{-1} B \tilde{K}_{x} \hat{x}+\eta v \hat{x}^{\top} \hat{A}^{\top} P A_{m}^{-1} \tilde{A} \hat{x} \\
& \leq-c_{1}\|e\|^{2}-c_{2}\left\|e_{p}\right\|^{2}+2 c_{3}\|e\|\left\|e_{p}\right\|-v c_{4}\|\hat{x}\|^{2}\left(\left\|\tilde{K}_{x}\right\|-c_{5}\right)^{2}+v c_{4} c_{5}^{2}\|\hat{x}\|^{2} \\
& -\eta c_{6}\|\hat{x}\|^{2}\left(\|\tilde{A}\|-c_{7}\right)^{2}+\eta c_{6} c_{7}^{2}\|\hat{x}\|^{2}
\end{aligned}
$$

where $\bar{R}=R-W \Delta A-\Delta A^{\top} W, c_{1}=\lambda_{\min }(Q), c_{2}=\lambda_{\min }(\bar{R}), c_{3}=\|P L C\|, c_{4}=\lambda_{\min }\left(B^{\top} A_{m}^{-\top} Q A_{m}^{-1} B\right), c_{5}=\frac{\left\|K_{x}^{* \top} B^{\top} P A_{m}^{-1} B\right\|}{c_{4}}$, $c_{6}=\lambda_{\min }\left(A_{m}^{-\top} Q A_{m}^{-1}\right), c_{7}=\frac{\left\|A^{\top} P A_{m}^{-1}\right\|}{c_{6}}$.

We utilize the inequality $2\|a\|\|b\| \leq\|a\|^{2}+\|b\|^{2}$ and also note that $\|\hat{x}\|^{2} \leq\|e\|^{2}+2\|e\|\left\|x_{m}\right\|+\left\|x_{m}\right\|^{2}$. Then, $\dot{V}\left(e, e_{p}, \tilde{K}_{x}, \tilde{A}\right)$ is bounded by

$$
\begin{aligned}
\dot{V}\left(e, e_{p}, \tilde{K}_{x}, \tilde{A}\right) \leq-\left(c_{1}-c_{3}-v c_{4} c_{5}^{2}-\eta c_{6} c_{7}^{2}\right)\|e\|^{2}+2\left(v c_{4} c_{5}^{2}+\eta c_{6} c_{7}^{2}\right)\|e\|\left\|x_{m}\right\|-\left(c_{2}-c_{3}\right)\left\|e_{p}\right\|^{2} \\
-v c_{4}\|\hat{x}\|^{2}\left(\left\|\tilde{K}_{x}\right\|-c_{5}\right)^{2}-\eta c_{6}\|\hat{x}\|^{2}\left(\|\tilde{A}\|-c_{7}\right)^{2}+\left(v c_{4} c_{5}^{2}+\eta c_{6} c_{7}^{2}\right)\left\|x_{m}\right\|^{2}
\end{aligned}
$$

Note that the ultimate bound of $\left\|x_{m}\right\|$ can be expressed as $\left\|x_{m}\right\| \leq c_{x} r_{0}$. Let $c_{8}=c_{1}-c_{3}-v c_{4} c_{5}^{2}-\eta c_{6} c_{7}^{2}, c_{9}=$ $\frac{\left(v c_{4} c_{5}^{2}+\eta c_{6} c_{7}^{2}\right) c_{x} r_{0}}{c_{8}}, c_{10}=c_{2}-c_{3}$, and $c_{11}=c_{8} c_{9}^{2}+\left(v c_{4} c_{5}^{2}+\eta c_{6} c_{7}^{2}\right) c_{x}^{2} r_{0}^{2}$. Then, $\dot{V}\left(e, e_{p}, \tilde{K}_{x}, \tilde{A}\right) \leq 0$ outside the compact set

$$
\begin{aligned}
\mathscr{S}=\left\{e(t) \in \mathbb{R}^{n}, e_{p}(t) \in \mathbb{R}^{n}, \tilde{K}_{x}(t) \in \mathbb{R}^{n \times m}, \tilde{A}(t) \in \mathbb{R}^{n \times n}: c_{8}\left(\|e\|-c_{9}\right)^{2}+c_{10}\left\|e_{p}\right\|^{2}\right. \\
\left.+v c_{4}\|\hat{x}\|^{2}\left(\left\|\tilde{K}_{x}\right\|-c_{5}\right)^{2}+\eta c_{6}\|\hat{x}\|^{2}\left(\|\tilde{A}\|-c_{7}\right)^{2} \leq c_{11}\right\}
\end{aligned}
$$

by choosing $L, Q, R, v$, and $\eta$ appropriately such that $c_{8}>0$ and $c_{10}>0$.

By setting $v=0$ and $\eta=0$, we recover the standard MRAC. Then, it can be shown by Barbalat's lemma that $\dot{V}\left(e, e_{p}, \tilde{K}_{x}, \tilde{A}\right)$ is uniformly continuous since $\ddot{V}\left(e, e_{p}, \tilde{K}_{x}, \tilde{A}\right)$ is bounded. It follows that $e(t) \rightarrow 0$ and $e_{p} \rightarrow 0$ as $t \rightarrow \infty$. 
Remark: If the reference model is not derived from the ideal controller of the non-SPR plant, the standard MRAC will not be able to stabilize the plant. On the other hand, the optimal control modification adaptive law can handle the mismatch between the plant and the reference model. Therefore, for a stable adaptation using the standard MRAC for output feedback adaptive control, the reference model must be established from the ideal controller design of the non-SPR plant. The mismatch between the reference model and the non-SPR plant causes MRAC to continue to seek a high-gain control in order to achieve asymptotic tracking. This would lead to instability.

Suppose a reference model is specified as

$$
\dot{x}_{m}=A_{m}^{*} x_{m}+B_{m} r
$$

where $A_{m}^{*}$ is not established from the non-SPR plant. Then, the model matching condition cannot be satisfied since there exists no solution of $K_{x}^{*}$. To show this, we see that suppose $K_{x}^{*}$ exists and can be solved using the pseudo-inverse of $B$ with $m<p<n$ as

$$
K_{x}^{*}=\left(B^{\top} B\right)^{-1} B^{\top}\left(A_{m}^{*}-A\right)
$$

But

$$
A+B K_{x}^{*}=A+B\left(B^{\top} B\right)^{-1} B^{\top}\left(A_{m}^{*}-A\right) \neq A_{m}^{*}
$$

For the standard MRAC, the tracking error equation in the presence of the mismatch between the reference model and the non-SPR plant is established as

$$
\dot{e}=A_{m}^{*} e+\left(A_{m}^{*}-A_{m}\right) \hat{x}-L C e_{p}-\tilde{A} \hat{x}-B \tilde{K}_{x} \hat{x}
$$

Because the optimal control modification only seeks bounded tracking, so the model matching condition is not satisfied. Using the linear asymptotic property of the optimal control modification, the asymptotic value of $K_{x}(t)$ and $\hat{A}(t)$ can be computed from Eqs. (93) and (94) by letting $\Gamma \rightarrow \infty$. Then, $K_{x}(t) \rightarrow \bar{K}_{x}$ and $\hat{A}(t) \rightarrow \bar{A}$ for a constant reference command signal. From the linear asymptotic property, we get

$$
\begin{gathered}
\bar{K}_{x} \hat{x}=-\frac{1}{v}\left(B^{\top} A_{m}^{*-\top} P B\right)^{-1} B^{\top} P e \\
\bar{A} \hat{x}=-\frac{1}{\eta} P^{-1} A_{m}^{* \top}\left(P e+W e_{p}\right)
\end{gathered}
$$

where $P=P^{\top}>0$ now solves the Lyapunov equation

$$
P A_{m}^{*}+A_{m}^{* \top} P=-Q
$$

We redefine the estimation errors as $\tilde{K}_{x}(t)=K_{x}(t)-\bar{K}_{x}$ and $\tilde{A}(t)=\hat{A}(t)-\bar{A}$. Then, the error equations are established as

$$
\begin{gathered}
\dot{e}=\left[A_{m}^{*}+\frac{1}{v} B\left(B^{\top} A_{m}^{*-\top} P B\right)^{-1} B^{\top} P+\frac{1}{\eta} P^{-1} A_{m}^{* \top} P\right] e-\left(L C-\frac{1}{\eta} P^{-1} A_{m}^{* \top} W\right) e_{p}+A_{m}^{*} \hat{x}-\tilde{A} \hat{x}-B \tilde{K}_{x} \hat{x} \\
\dot{e}_{p}=\frac{1}{\eta} P^{-1} A_{m}^{* \top} P e+\left(A_{p}+\Delta A+\frac{1}{\eta} P^{-1} A_{m}^{* \top} W\right) e_{p}+A \hat{x}-\tilde{A} \hat{x}
\end{gathered}
$$

The instability of the standard MRAC and the bounded tracking of the closed-loop system with the optimal control modification can be stated in the following theorem:

Theorem 6: The standard MRAC results in instability due to the mismatch between the SPR reference model and the non-SPR plant if $A+B K_{x}^{*}=A_{m} \neq A_{m}^{*}$ and $P A_{m}+A_{m}^{\top} P \nless 0$, whereas a stable adaptation of the plant can be achieved with the optimal control modification.

Proof: Choose the same Lyapunov candidate function in Eq. (97). Then, for the standard MRAC, $\dot{V}\left(e, e_{p}, \tilde{K}_{x}, \tilde{A}\right)$ is evaluated as

$$
\dot{V}\left(e, e_{p}, \tilde{K}_{x}, \tilde{A}\right)=e^{\top}\left(P A_{m}^{*}+A_{m}^{* \top} P\right) e+e^{\top} P\left(A_{m}^{*}-A_{m}\right) \hat{x}+\hat{x}^{\top}\left(A_{m}^{* \top}-A_{m}^{\top}\right) P e-2 e^{\top} P L C e_{p}-e_{p}^{\top} \bar{R} e_{p}
$$


Upon substituting $\hat{x}(t)=x_{m}(t)-e(t)$, we get

$$
\dot{V}\left(e, e_{p}, \tilde{K}_{x}, \tilde{A}\right)=e^{\top}\left(P A_{m}+A_{m}^{\top} P\right) e+2 e^{\top} P\left(A_{m}^{*}-A_{m}\right) x_{m}-2 e^{\top} P L C e_{p}-e_{p}^{\top} \bar{R} e_{p}
$$

Note that $P A_{m}+A_{m}^{\top} P$ is not necessarily negative definite. Therefore,

$$
\dot{V}\left(e, e_{p}, \tilde{K}_{x}, \tilde{A}\right) \leq\left\|P A_{m}+A_{m}^{\top} P\right\|\|e\|^{2}+\left\|P\left(A_{m}^{*}-A_{m}\right)\right\|\left(\|e\|^{2}+\left\|x_{m}\right\|^{2}\right)+c_{3}\left(\|e\|^{2}+\left\|e_{p}\right\|^{2}\right)-c_{2}\left\|e_{p}\right\|^{2}
$$

Thus, $\dot{V}\left(e, e_{p}, \tilde{K}_{x}, \tilde{A}\right) \not \leq 0$. Therefore, the tracking error is unbounded and the closed-loop system is unstable. On the other hand, for the optimal control modification, $\dot{V}\left(e, e_{p}, \tilde{K}_{x}, \tilde{A}\right)$ is evaluated as

$$
\begin{array}{r}
\dot{V}\left(e, e_{p}, \tilde{K}_{x}, \tilde{A}\right)=e^{\top}\left\{-\frac{1}{\eta} Q+\frac{1}{v} P B\left[\left(B^{\top} A_{m}^{*-\top} P B\right)^{-1}+\left(B^{\top} A_{m}^{*-\top} P B\right)^{-\top}\right] B^{\top} P\right\} e^{\top}+2 e^{\top} P A_{m}^{*} x_{m} \\
-2 e^{\top}\left[P L C-\frac{1}{\eta}\left(A_{m}^{* \top}+P A_{m}^{*} P^{-1}\right) W+A^{\top} W\right] e_{p}+e_{p}^{\top}\left[-\bar{R}+\frac{1}{\eta} W\left(P^{-1} A_{m}^{* \top}+A_{m}^{*} P^{-1}\right) W\right] e_{p} \\
+2 e_{p}^{\top} W A x_{m}+2 v \hat{x}^{\top} K_{x}^{\top} B^{\top} P A_{m}^{-1} B \tilde{K}_{x} \hat{x}+\eta v \hat{x}^{\top} \hat{A}^{\top} P A_{m}^{-1} \tilde{A} \hat{x}
\end{array}
$$

Note that $P^{-1} A_{m}^{* \top}+A_{m}^{*} P^{-1}=-P^{-1} Q P^{-1}$. Then,

$$
\begin{aligned}
\dot{V}\left(e, e_{p}, \tilde{K}_{x}, \tilde{A}\right) \leq-c_{12}\|e\|^{2}-c_{13}\left\|e_{p}\right\|^{2}+2 c_{14}\|e\|\left\|e_{p}\right\| & +2 c_{15}\|e\|+2 c_{16}\left\|e_{p}\right\|-v c_{4}\|\hat{x}\|^{2}\left(\left\|\tilde{K}_{x}\right\|-c_{5}\right)^{2} \\
& +v c_{4} c_{5}^{2}\|\hat{x}\|^{2}-\eta c_{6}\|\hat{x}\|^{2}\left(\|\tilde{A}\|-c_{7}\right)^{2}+\eta c_{6} c_{7}^{2}\|\hat{x}\|^{2}
\end{aligned}
$$

where $c_{12}=\lambda_{\min }\left(\frac{1}{\eta} Q-\frac{1}{v} P B\left[\left(B^{\top} A_{m}^{*-\top} P B\right)^{-1}+\left(B^{\top} A_{m}^{*-\top} P B\right)^{-\top}\right] B^{\top} P\right), c_{13}=\lambda_{\min }\left(\bar{R}-\frac{1}{\eta} W P^{-1} Q P^{-1} W\right), c_{14}=$ $\left\|P L C+\frac{1}{\eta} Q P^{-1} W+A^{\top} W\right\|, c_{15}=\left\|P A_{m}^{*}\right\| c_{x} r_{0}, c_{16}=\|W A\| c_{x} r_{0}$, and $c_{4,5,6,7}$ are defined previously.

Further simplification using $\|\hat{x}\|^{2} \leq\|e\|^{2}+2\|e\|\left\|x_{m}\right\|+\left\|x_{m}\right\|^{2}$ leads to

$$
\dot{V}\left(e, e_{p}, \tilde{K}_{x}, \tilde{A}\right) \leq-c_{17}\left(\|e\|-c_{18}\right)^{2}-c_{19}\left(\left\|e_{p}\right\|-c_{20}\right)^{2}-v c_{4}\|\hat{x}\|^{2}\left(\left\|\tilde{K}_{x}\right\|-c_{5}\right)^{2}-\eta c_{6}\|\hat{x}\|^{2}\left(\|\tilde{A}\|-c_{7}\right)^{2}
$$

where $c_{17}=c_{12}-c_{14}-v c_{4} c_{5}^{2}-\eta c_{6} c_{7}^{2}, c_{18}=\frac{c_{15}+\left(v c_{4} c_{5}^{2}+\eta c_{6} c_{7}^{2}\right) c_{x} r_{0}}{c_{17}}, c_{19}=c_{13}-c_{14}, c_{20}=\frac{c_{16}}{c_{19}}$, and $c_{21}=c_{17} c_{18}^{2}+$ $c_{19} c_{20}^{2}+\left(v c_{4} c_{5}^{2}+\eta c_{6} c_{7}^{2}\right) c_{x}^{2} r_{0}^{2}$.

Choose $L, Q, R, v$, and $\eta$ such that $c_{17}>0$ and $c_{19}>0$. Then, $\dot{V}\left(e, e_{p}, \tilde{K}_{x}, \tilde{A}\right) \leq 0$ outside a compact set. Therefore, the closed-loop system is uniformly ultimately bounded with the optimal control modification.

Using the linear asymptotic property of the optimal control modification, $L, Q, v$ and $\eta$ can also be chosen such that the asymptotic closed-loop matrix formed by $(\dot{x}(t), \dot{\hat{x}}(t))$

$$
A_{c}=\left[\begin{array}{cc}
A & \frac{1}{v} B\left(B^{\top} A_{m}^{*-\top} P B\right)^{-1} B^{\top} P \\
L C-\frac{1}{\eta} P^{-1} A_{m}^{* \top} W & -L C+\frac{1}{v} B\left(B^{\top} A_{m}^{*-\top} P B\right)^{-1} B^{\top} P+\frac{1}{\eta} P^{-1} A_{m}^{* \top}(P+W)
\end{array}\right]
$$

is Hurwitz.

To demonstrate the observer state feedback adaptive control, we return to Example 1.

Example 3: The non-minimum phase plant in Example 1 is defined by the following matrices:

$$
A=\left[\begin{array}{cc}
a & g \\
0 & -1
\end{array}\right], B=\left[\begin{array}{l}
b \\
1
\end{array}\right], C=\left[\begin{array}{ll}
1 & 0
\end{array}\right]
$$

where $a=-2, b=1$, and $g=-2$. 
The Kalman filter gain is computed with $\bar{A}=\left[\begin{array}{cc}0 & g \\ 0 & -1\end{array}\right]$ as

$$
L=\left[\begin{array}{c}
1.5440 \\
-0.3460
\end{array}\right]
$$

Using $Q=100$ and $R=1$, the LQR control gains are obtained as

$$
K_{x}^{*}=\left[\begin{array}{ll}
-2.7327 & -5.4654
\end{array}\right], k_{r}=-9.8058
$$

Choose $\Gamma_{x}=I, \Gamma_{A}=0.1 I, Q=I$, and $R=I$. Figure 6 shows the closed-loop response with the standard MRAC of the plant output $y(t)$ which tracks the redesigned reference model very well. Notice that the reference model now has a non-minimum phase behavior as evidenced by the reversal in the initial response. All the control and adaptive parameter signals are bounded.

Suppose, instead of the reference model computed from the LQR, we use the ideal minimum phase first-order reference model described by the following matrices:

$$
A_{m}^{*}=\left[\begin{array}{cc}
a_{m} & 0 \\
0 & -1
\end{array}\right], B=\left[\begin{array}{c}
b_{m} \\
0
\end{array}\right]
$$

Figure 7 shows the unstable closed-loop response with the standard MRAC which no longer tracks the reference model. The adaptive parameters are unbounded and drifting as $t \rightarrow \infty$.

The optimal control modification is then used with $v=\eta=0.08$ selected. Figure 8 shows the closed-loop response with the optimal control modification which is able to track the ideal minimum phase reference model very well. All the control and adaptive parameter signals are bounded.
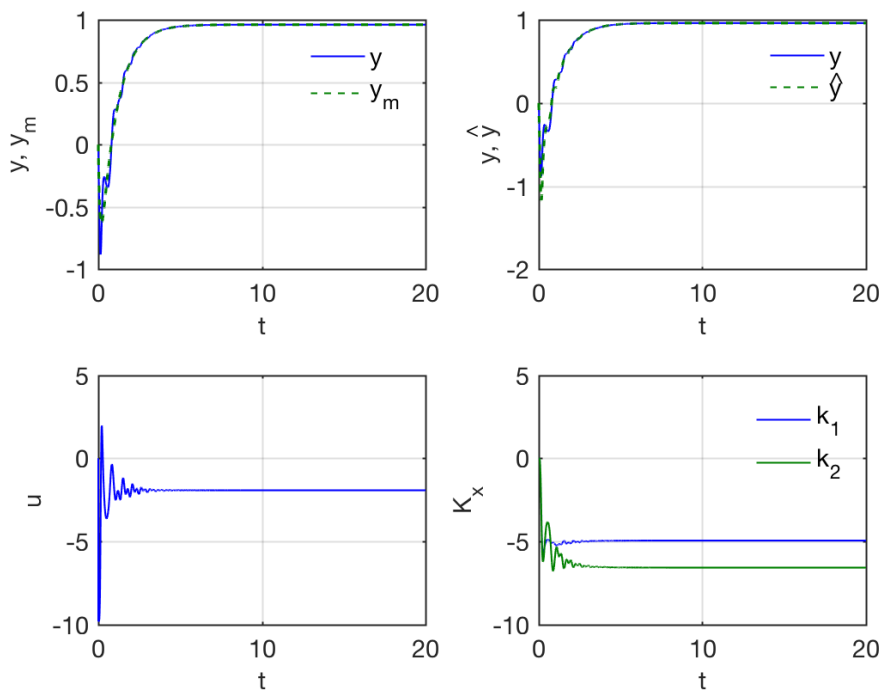

Figure 6: Closed-Loop Output Response to LQR Non-Minimum Phase Reference Model with Observer State Feedback MRAC 

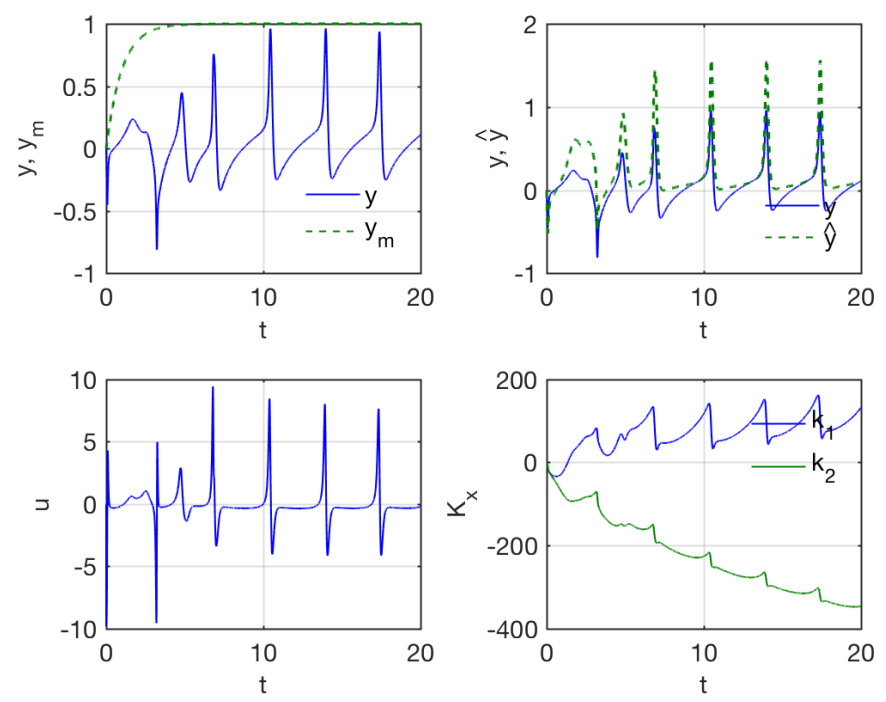

Figure 7: Closed-Loop Output Response to Ideal Minimum Phase Reference Model with Observer State Feedback MRAC
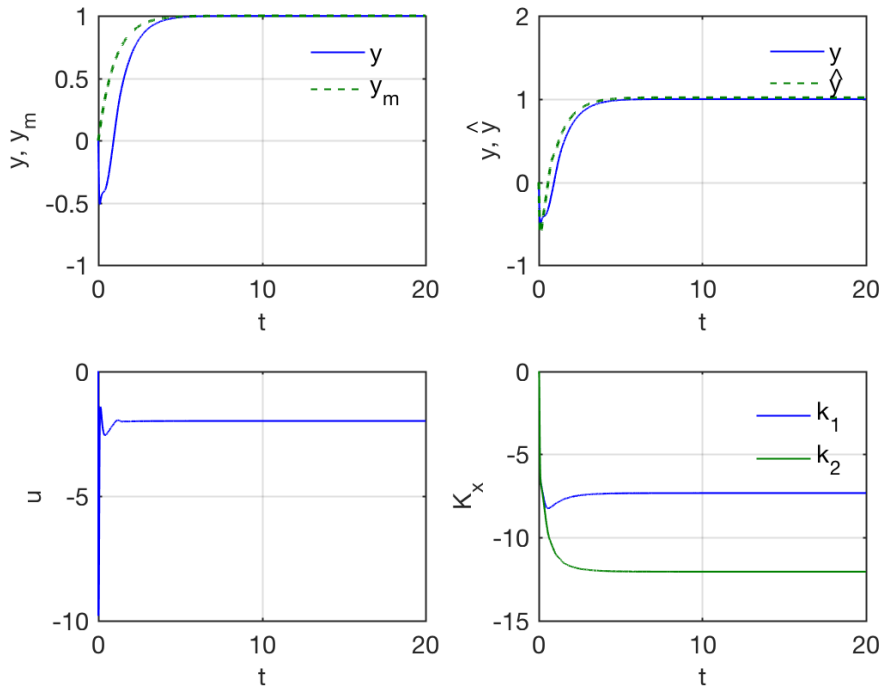

Figure 8: Closed-Loop Output Response to Ideal Minimum Phase Reference Model with Optimal Control Modification

This example illustrates the significance of the reference model in adaptive control of non-minimum phase plants. If the reference model can be redesigned to fully account for the non-minimum phase behavior, then the standard MRAC can achieve asymptotic tracking, albeit with a non-minimum phase behavior. Otherwise, even with the observer-based output feedback adaptive control design, if the reference model is SPR, instability will still result 
with the standard MRAC. On the other hand, the optimal control modification can produce bounded tracking for both types of reference models.

\section{Application of Flexible Wing Aircraft}

Consider a flexible wing Generic Transport Model (GTM) equipped with distributed wing shaping control surfaces, called Variable Camber Continuous trailing edge flaps (VCCTEF). The VCCTEF are multi-functional flight control surfaces designed for wing shaping control for drag minimization, flutter suppression, and load alleviation. The redundancy of the VCCTEF enables a multi-objective flight control capability which provides the traditional flight control functions in roll, pitch, and yaw in concert with the wing shaping control objectives such as drag minimization and gust load alleviation.

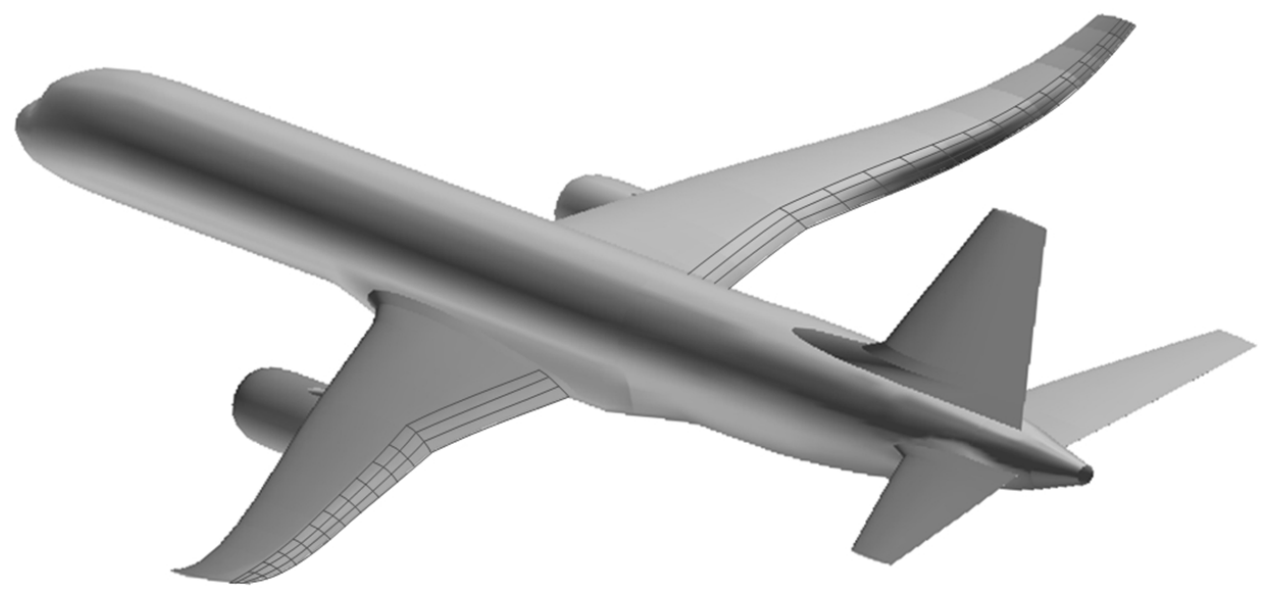

Figure 9: GTM with with Variable Camber Continuous Trailing Edge Flap

Consider a linearized model of a flexible aircraft with an uncertainty in the rigid aircraft states

$$
\begin{aligned}
\dot{x}=(A+\Delta A) x+B u & \Leftrightarrow\left[\begin{array}{c}
\dot{x}_{r} \\
\dot{x}_{e}
\end{array}\right]=\left[\begin{array}{cc}
A_{r r}+\Delta A_{r r} & A_{r e} \\
A_{e r} & A_{e e}
\end{array}\right]\left[\begin{array}{c}
x_{r} \\
x_{e}
\end{array}\right]+\left[\begin{array}{c}
B_{r} \\
B_{e}
\end{array}\right] u \\
y & =C x \Leftrightarrow y=\left[\begin{array}{ll}
I & 0
\end{array}\right]\left[\begin{array}{c}
x_{r} \\
x_{e}
\end{array}\right]=x_{r}
\end{aligned}
$$

where $x(t) \in \mathbb{R}^{n}$ is a state vector that comprises a rigid aircraft state vector $x_{r}(t) \in \mathbb{R}^{p}$ and an elastic wing state vector $x_{e}(t) \in \mathbb{R}^{n-p}, u(t) \in \mathbb{R}^{m}$ is a control vector, $A \in \mathbb{R}^{n \times n}$ and $B \in \mathbb{R}^{n \times m}$ are constant and known matrices, and $\Delta A \in \mathbb{R}^{n \times n}$ is an uncertainty. The open-loop plant is stable with $A$ Hurwitz. The output vector $y(t)$ is the rigid aircraft state vector $x_{r}(t)$ is accessible for feedback. The rigid aircraft state vector $x_{r}(t)=\left[\begin{array}{lll}\alpha(t) & q(t) & \theta(t)\end{array}\right]^{\top}$ comprises the angle of attack $\alpha(t)$, pitch rate $q(t)$, and pitch attitude $\theta(t)$.

The control vector $u(t)$ comprises the elevator deflection and 16 individual trailing edge flap segments, each connected to an adjacent flap by an elastomer transition section as shown in blue in Fig. 10. For clarity, the trailing edge flap segment is the outermost segment of the three cambered segments that form each spanwise flap section. This segment is considered a fast-acting flight control surface [?] whereas the other two segments are slow-acting flight control surfaces for drag reduction. Due to the stiffness imposed by the elastomer transition section, a constraint on the relative deflection of any two adjacent flaps is imposed on the control input command. In order to address this relative deflection constraint, a virtual control concept is used whereby the flap deflection is mapped into a mathematically smooth shape function whose coefficients are the virtual control variables [?]. A cubic Chebyshev polynomial is selected as a candidate shape function. Then, the flap deflection of the $i$-th flap is expressed as

$$
\delta_{i}=c_{0}+c_{1} k+c_{2}\left(2 k^{2}-1\right)+c_{3}\left(4 k^{3}-3 k\right)
$$


where $k=\frac{i-1}{n-1}, i=1,2, \ldots, 16, n=16$ with $\delta_{1}$ being the inboard flap, and $c_{j}(t), j=0,1,2,3$ are the virtual control variables. Thus, the control vector is represented by $u(t)=\left[\begin{array}{llll}c_{0}(t) & c_{1}(t) & c_{2}(t) & c_{3}(t)\end{array}\right]^{\top}$.

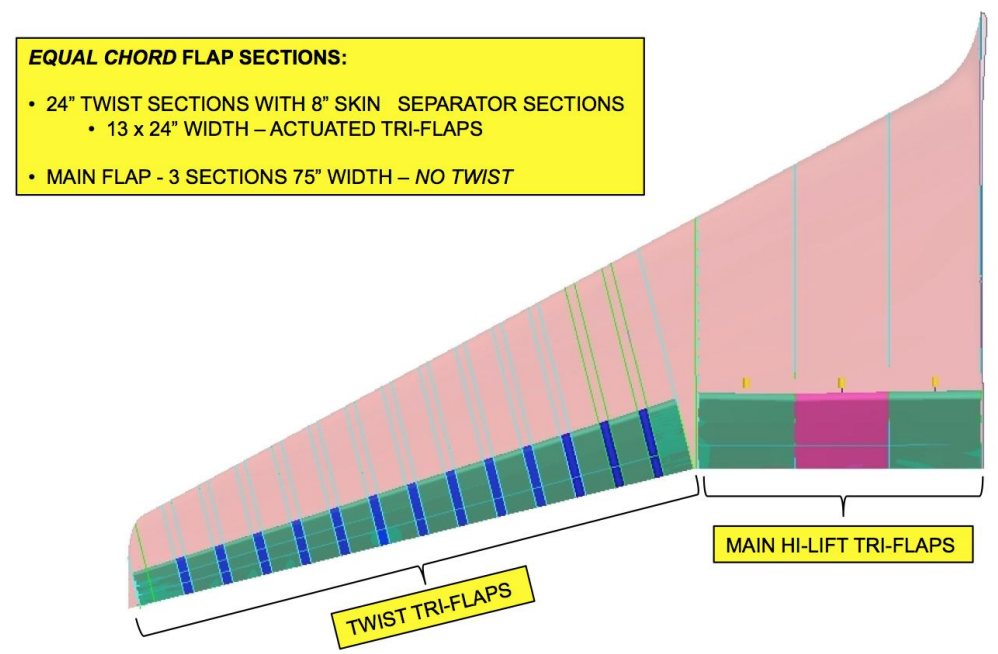

Figure 10: Variable Camber Continuous Trailing Edge Flap (VCCTEF)

\subsection{Output Feedback Design}

A step command of the pitch attitude is specified as $r(t)=\theta_{c}(t)$. A nominal LQR controller is designed as

$$
u_{\text {nom }}=K_{x} x+K_{r} r
$$

where $K_{x}=-R^{-1} B_{r}^{\top} W, K_{r}=-R^{-1} B_{r}^{\top}\left(A_{r r}^{\top}-W B_{r} R^{-1} B_{r}^{\top}\right)^{-1} I_{\theta}^{\top} Q, I_{\theta}=\left[\begin{array}{lll}0 & 0 & 1\end{array}\right]$, and $W$ solves the Riccati equation+

$$
W A_{r r}+A_{r r}^{\top} W-W B_{r} R^{-1} B_{r}^{\top} W+I_{\theta}^{\top} Q I_{\theta}=0
$$

The reference model is then established as

$$
\dot{y}_{m}=A_{r r} y+B_{r} u_{n o m}=A_{m} y_{m}+B_{m} r
$$

where $A_{m}=A_{r r}+B_{r} K_{x}$ and $B_{m}=B_{r} K_{r}$.

In the presence of the uncertainty $\Delta A$, the ideal controller is designed as

$$
u^{*}=B_{r}^{\top} I_{r}^{\top}\left(I_{r} B_{r} B_{r}^{\top} I_{r}^{\top}\right)^{-1} I_{r}\left[A_{m} y+B_{m} r-\left(A_{r r}+\Delta A_{r r}\right) y-A_{r e} \Phi_{y}-A_{r e} \Phi_{u}\right]
$$

where $I_{r}=\left[\begin{array}{ccc}1 & 0 & 0 \\ 0 & 1 & 0\end{array}\right]$. The output feedback adaptive controller is then designed as

$$
u=-\left(C_{1} B_{1}\right)^{+}\left[\left(A_{r r}-A_{m}\right) y-B_{m} r+\Theta^{\top} \Phi(y, u)\right]
$$

where $\left(C_{1} B_{1}\right)^{+}=B_{r}^{\top} I_{r}^{\top}\left(I_{r} B_{r} B_{r}^{\top} I_{r}^{\top}\right)^{-1} I_{r}, \Phi(y, u)=\left[\begin{array}{lll}y^{\top}(t) & \Phi_{y}^{\top}(t) & \Phi_{u}^{\top}(t)\end{array}\right]^{\top}$, and $\Theta(t)$ is computed by the optimal control modification update law

$$
\dot{\Theta}=-\Gamma \Phi(y, u)\left[e_{y}^{\top} P-v \Phi^{\top}(y, u) \Theta P A_{m}^{-1}\right]
$$

For simulations, we choose the plant model for the GTM with 50\% fuel loading at Mach 0.8 and an altitude of $36,000 \mathrm{ft}$ with the first four elastic modes. The plant model contains 27 states: 3 rigid-body states corresponding to 
$x_{r}(t)$ and 24 elastic states for the four elastic modes. The state-space representation for each elastic mode includes a generalized displacement, a generalized speed, two unsteady aerodynamic lag states for the generalized displacement, and two unsteady aerodynamic lag states for the generalized speed. $\Delta A_{r r}$ is modeled as a $20 \%$ reduction in $C_{L_{\alpha}}, C_{L_{q}}$, $C_{m_{\alpha}}$, and $C_{m_{q}}$. The nominal controller is designed with $Q=10$ and $R=I$. This results in the following reference model matrix:

$$
A_{m}=\left[\begin{array}{ccc}
-0.4567 & 0.8971 & -0.2197 \\
-0.2603 & -3.6750 & -8.2325 \\
0 & 1 & 0
\end{array}\right]
$$

which yields the eigenvalues $-1.8491 \pm 2.2632 i$ and -0.4335 .

The modification parameter $v$ is chosen to be $v=1$ which stabilizes the asymptotic closed-loop matrix

$$
A_{c}=\left[\begin{array}{cc}
A_{m}+\frac{1}{v} B_{r}\left(C_{1} B_{1}\right)^{+} P^{-1} A_{m} P & A_{12} \\
A_{e r}+\frac{1}{v} B_{e}\left(C_{1} B_{1}\right)^{+}\left(A_{m}-A_{r r}+P^{-1} A_{m} P\right) & A_{22}
\end{array}\right]
$$

The adaptation rate matrix is chosen to be $\Gamma=10^{4} I$. The matrix $P$ is computed from the Lyapunov equation using $Q=I$. The closed-loop response of the aircraft due to the output feedback adaptive controller with the optimal control modification is shown in Fig. 11. The response is completely stable. After the first doublet, the pitch attitude tracking is improved.
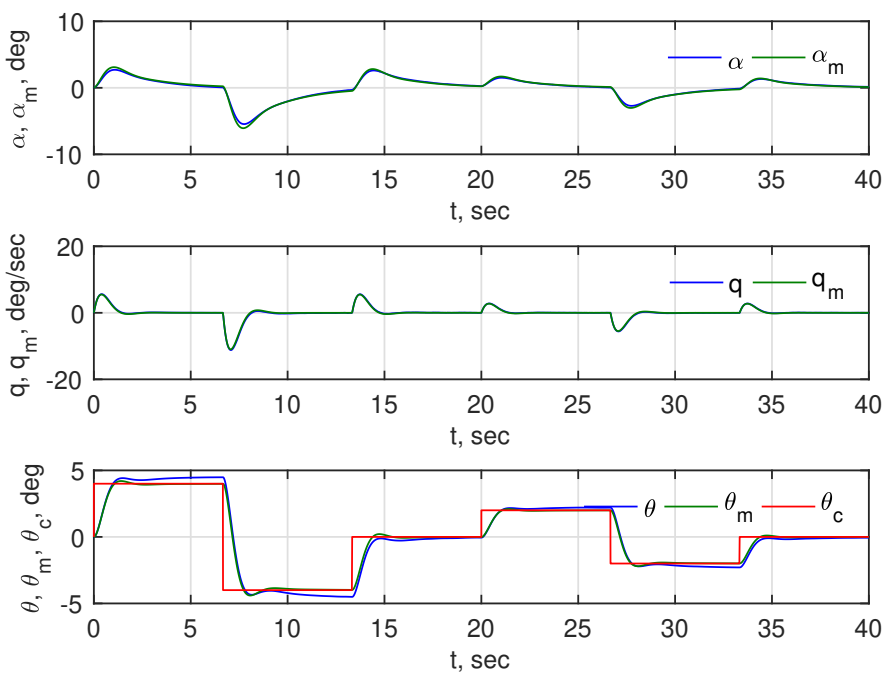

Figure 11: Closed-Loop Response of Aircraft to Output Feedback Adaptive Control with Optimal Control Modification

On the other hand, the standard MRAC version of the output feedback adaptive controller can only be stabilized for a very small value of the adaptation rate matrix. The closed-loop response of the aircraft due to the standard MRAC output feedback adaptive controller with $\Gamma=10^{-3} I$ is shown in Fig. 12. The pitch attitude response is seen to be better, but the angle of attack response is slightly worse than that with the optimal control modification. Also, the standard MRAC is highly sensitive to the adaptation rate matrix and cannot tolerate a larger value of $10^{-3} I$. In contrast, the optimal control modification can tolerate a much larger value of the adaptation rate matrix. Because of the presence of the damping term in the optimal control modification adaptive law, an implicit integration can be implemented. This permits the adaptation rate matrix to be set as high as $10^{14} I$ in the simulations. 

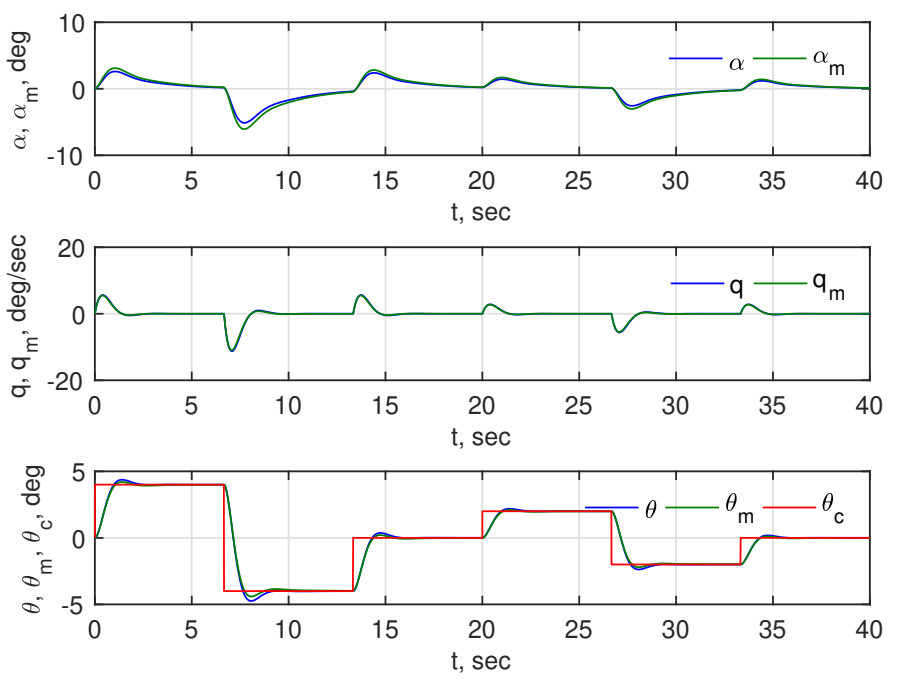

Figure 12: Closed-Loop Response of Aircraft to Output Feedback Adaptive Control with Standard MRAC

\subsection{Observer-Based Design}

The observer state-space model of the plant is constructed as

$$
\dot{\hat{x}}=(A+\Delta \hat{A}) \hat{x}+L(y-\hat{y})+B u
$$

where $\Delta \hat{A}(t)$ is estimated by the following adaptive law:

$$
\Delta \dot{\hat{A}}^{\top}=\Gamma_{A} \hat{x}\left(e^{\top} P+e_{p}^{\top} W+\eta \hat{x}^{\top} \Delta \hat{A}^{\top} P A_{m}^{-1}\right)
$$

The nominal controller is designed as

$$
u_{\text {nom }}=K_{x} \hat{x}+K_{r} r
$$

where $K_{x}$ and $K_{r}$ are computed from the LQR solution for the plant model.

The observer-based output feedback adaptive controller is then designed as

$$
u=u_{n o m}+\Delta K_{x} \hat{x}
$$

where $\Delta K_{x}$ is computed from the following adaptive law:

$$
\Delta \dot{K}_{x}^{\top}=\Gamma_{x} \hat{x}\left(e^{\top} P+v \hat{x}^{\top} \Delta K^{\top} B^{\top} P A_{m}^{-1}\right) B
$$

Figure 13 shows the closed-loop response of the aircraft to the observer-based output feedback adaptive controller with the optimal control modification using $v=108$ which stabilizes the closed-loop plant for $\Gamma_{x}=10^{4} I$ and without $\Delta \hat{A}(t)$ estimated. The $P$ and $W$ matrices are computed from the Lyapunov equations using $Q=R=I$. The nominal $\mathrm{LQR}$ controller is designed with $Q=10$ and $R=I$. The Kalman filter observer is designed using $Q=R=G=I$. The tracking performance of the pitch attitude with the observer-based output feedback adaptive controller is worse than that with the output feedback adaptive controller. 

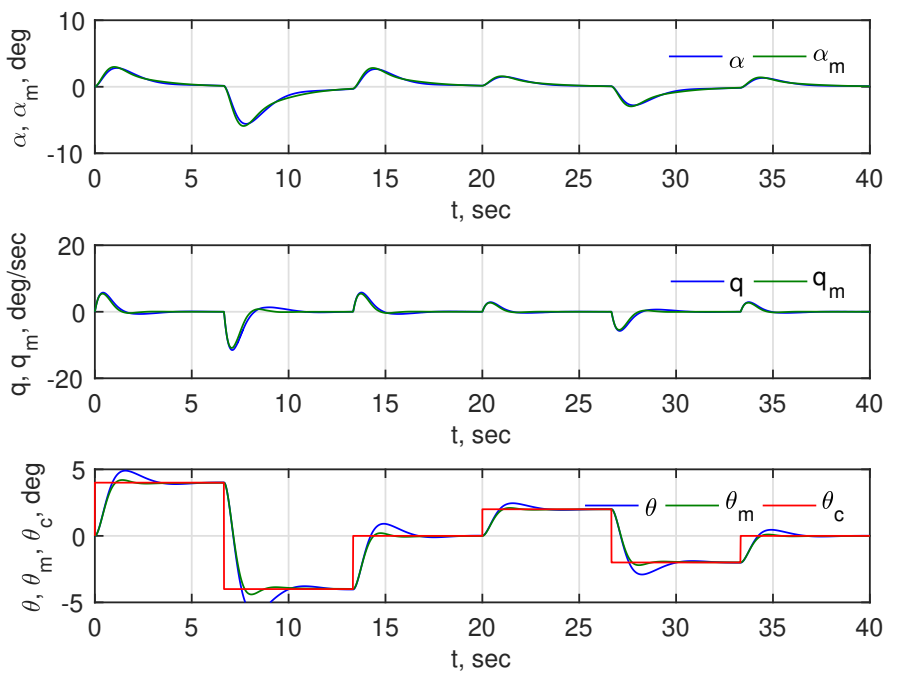

Figure 13: Closed-Loop Response of Aircraft to Output Feedback Adaptive Control with Optimal Control Modification

In contrast, the standard MRAC version of the observer-based output feedback adaptive controller performs worse than the optimal control modification. The adaptation rate matrix for the standard MRAC is highly sensitive and can only be set to a small value. In the simulations, $\Gamma_{x}=10^{-4}$ is chosen for the standard MRAC. The pitch attitude tracking is much worse with the standard MRAC than with the optimal control modification, as shown in Fig. 14. The pitch rate response shows a high frequency signature.
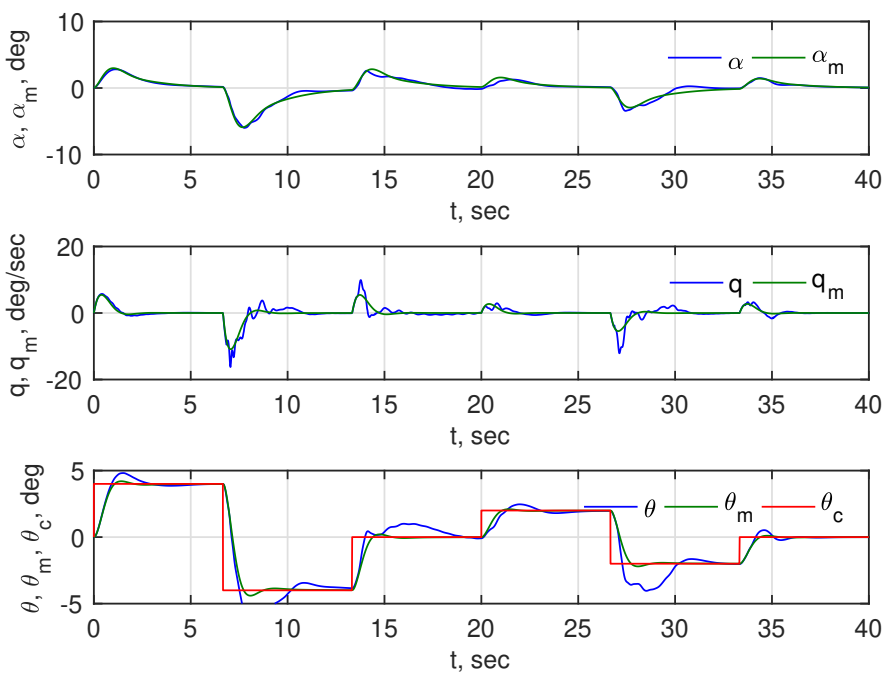

Figure 14: Closed-Loop Response of Aircraft to Output Feedback Adaptive Control with Standard MRAC

Examining the norm of the $P$ matrix, it is seen that $\|P\|=1.9322 \times 10^{5}$. In comparison, for the output feedback adaptive controller design, $\|P\|=2.8521$. The large value of the $P$ matrix thus effectively prevents the adaptation 
rate matrix for the standard MRAC from being set to a reasonable value. A simulation of the standard MRAC with the normalized $P$ matrix is implemented. The normalized $P$ matrix is obtained by dividing the computed $P$ matrix from the Lyapunov equation using $Q=I$ by its norm. This allows the adaptation rate matrix to increase to $\Gamma_{x}=10$. The closed-loop response to the standard MRAC is improved with the normalized $P$ matrix, as shown in Fig. 15 . Comparing to the optimal control modification, the closed-loop response to the standard MRAC with the normalized $P$ matrix appears slightly worse since high frequency contents in the pitch rate can still be seen.
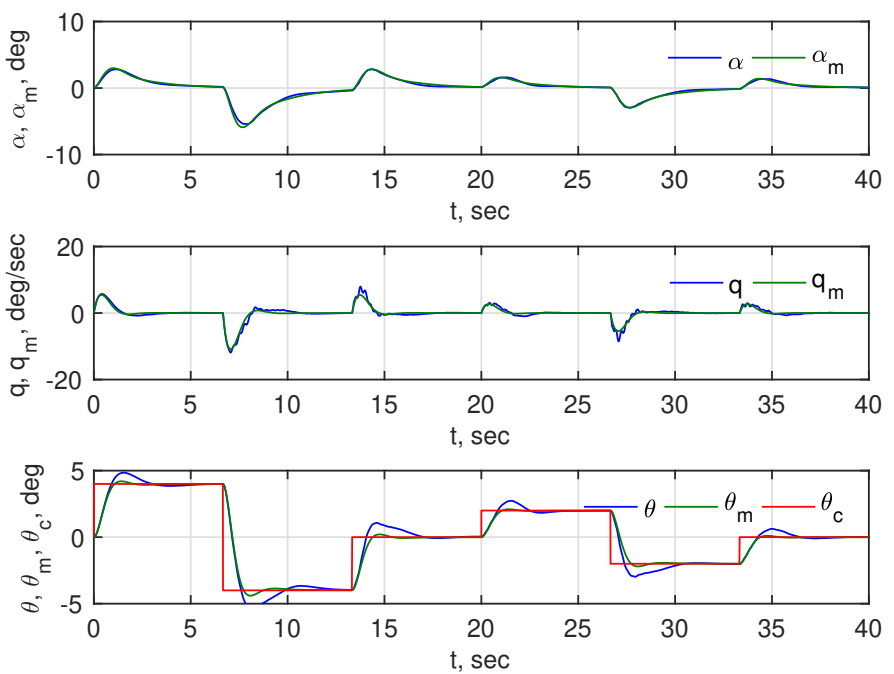

Figure 15: Closed-Loop Response of Aircraft to Output Feedback Adaptive Control with Standard MRAC Using Normalized $P$ Matrix
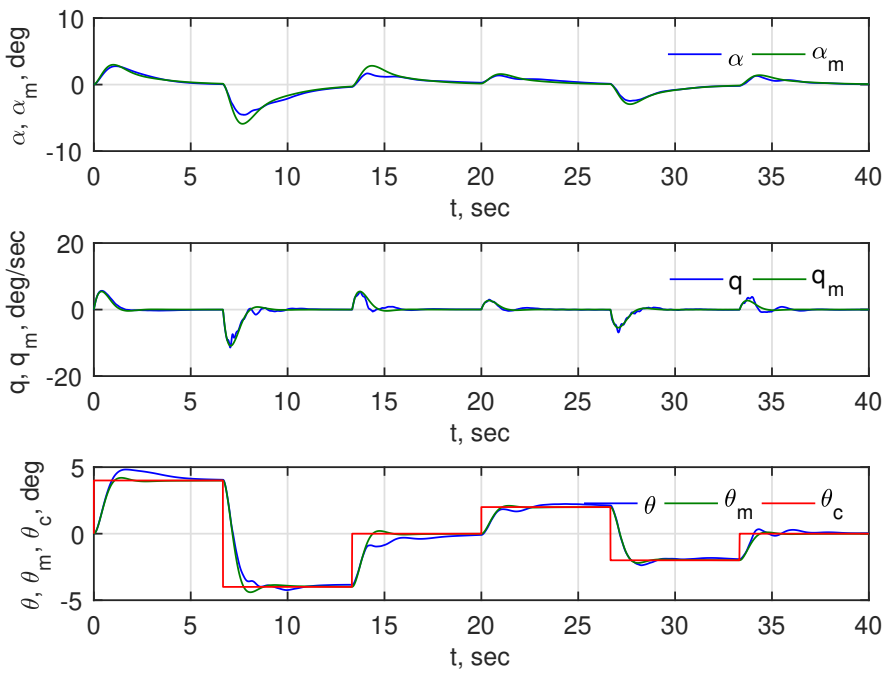

Figure 16: Closed-Loop Response of Aircraft to Output Feedback Adaptive Control with Standard MRAC and $\Delta \hat{A}(t)$ Estimation Using Normalized $P$ and $W$ Matrices 
Figure 16 is the closed-loop response to the standard MRAC with the normalized $P$ matrix and $\Delta \hat{A}(t)$ estimated in the observer design using $\Gamma_{A}=10$ with the normalized $W$ matrix. The pitch attitude tracking is much improved over that without the $\Delta \hat{A}(t)$ estimation. The angle of attack response, however, is worsened. Comparing to the output feedback adaptive controller with the optimal control modification, the closed-loop response appears to be slightly worse.

\section{Conclusions}

An output feedback adaptive control method based on the optimal control modification is presented. The method is shown to be able to stabilize SISO systems with relative degree 1 by taking advantage of the linear asymptotic property. This method is extended to non-SPR MIMO systems. A stability condition is established to ensure that the standard MRAC can achieve a stable output feedback adaptation. When this stability condition is not met, the optimal control modification can still achieve a stable adaptation, but the standard MRAC results in instability. The potential degraded tracking performance of the output feedback adaptive control for non-minimum phase systems can be addressed by observer-based output feedback adaptive control using the Luenberger observer design. The optimal control modification demonstrates the ability to achieve a stable adaptation for observer-based output feedback adaptive control in the presence of the mismatch between the non-SPR plant and the SPR reference model which otherwise causes instability for the standard MRAC. Simulations for a flexible wing aircraft demonstrate the effectiveness of the optimal control modification for output feedback adaptive control.

\section{References}

[1] Åström, K. J. and Wittenmark B., Adaptive Control, Dover Publications, Inc., 2008.

[2] Ioannu, P. A. and Sun, J., Robust Adaptive Control, Prentice-Hall, Inc., 1996.

[3] Narendra, K. S. and Annaswamy, A. M., Stable Adaptive Systems, Dover Publications, 2005.

[4] Nguyen, N., "Adaptive Control for Linear Uncertain Systems with Unmodeled Dynamics Revisited via Optimal Control Modification," AIAA Guidance, Navigation, and Control Conference, AIAA-2013-4988, August 2013.

[5] Nguyen, N., "Optimal Control Modification for Robust Adaptive Control with Large Adaptive Gain," Systems \& Control Letters, 61 (2012) pp. 485-494.

[6] Lavretsky, E. and Wise, K., Robust and Adaptive Control, Springer, 2012.

[7] Lavretsky, E., "Robust and Adaptive Output Feedback Control for Non-Minimum Phase Systems with Arbitrary Relative Degree," AIAA Guidance, Navigation, and Control Conference, AIAA-2017-1490, January 2017.

[8] Slotine, J. -J. and Li, W., Applied Nonlinear Control, Prentice-Hall, Inc., 1991.

[9] Hsu, L., Teixeira, M. C. M., Costa, R. R., and Assunc̃ao, E., "Necessary and Sufficient Condition for Generalized Passivity, Passification and Application to Multivariable Adaptive Systems," 18th International Federation of Automatic Control World Congress, August 2011. 